\title{
FINITE ELEMENT HETEROGENEOUS MULTISCALE METHOD FOR THE WAVE EQUATION: LONG-TIME EFFECTS*
}

\author{
ASSYR ABDULLE ${ }^{\dagger}$, MARCUS J. GROTE ${ }^{\ddagger}$, AND CHRISTIAN STOHRER ${ }^{\S}$
}

\begin{abstract}
A new finite element heterogeneous multiscale method (FE-HMM) is proposed for the numerical solution of the wave equation over long times in a rapidly varying medium. Our new FE-HMM-L method captures not only the short-time behavior of the wave field, well described by classical homogenization theory, but also more subtle long-time dispersive effects, both at a computational cost independent of the microscale. Optimal error estimates in the energy norm and the $L^{2}$-norm are proved over finite time intervals, which imply convergence to the solution from classical homogenization theory when both the macro- and the microscale are refined simultaneously. Numerical experiments illustrate the usefulness of the FE-HMM-L method and corroborate the theory.
\end{abstract}

Key words. multiscale method, heterogeneous media, numerical homogenization, wave equation, second-order hyperbolic problems, long-time behavior, dispersive waves

AMS subject classifications. Primary, 65M60, 65N30; Secondary, 74Q10, 74Q15, 35L05

DOI. $10.1137 / 13094195 \mathrm{X}$

1. Introduction. Wave propagation across heterogeneous media, whether manmade or natural, is ubiquitous throughout scientific and engineering applications. When heterogeneities occur everywhere, and at a microscopic scale $\varepsilon$ much smaller than the scales of interest, standard numerical methods become prohibitively expensive. Indeed classical finite difference (FD) methods or finite element methods (FEMs) require grid resolution down to the finest scale in the medium, even when the typical wave length occurs at the macroscopic scale. In contrast, homogenization theory yields properly averaged equations that capture the essential effects of the rapidly varying medium in the limit $\varepsilon \rightarrow 0[12,13,18]$. Since these homogenized equations are explicitly available only in very few situations, such as periodic or random stationary fields, numerical multiscale methods that overcome these limitations are needed.

For wave phenomena in strongly heterogeneous media, the wave equation

$$
\partial_{t t} u^{\varepsilon}-\nabla \cdot\left(a^{\varepsilon} \nabla u^{\varepsilon}\right)=F
$$

with a rapidly varying coefficient, $a^{\varepsilon}(x)$, often serves as a model. Here $\varepsilon$ represents a small scale in the problem, $0<\varepsilon \ll 1$, which characterizes the multiscale nature of the tensor $a^{\varepsilon}$. In the limit $\varepsilon \rightarrow 0$, classical homogenization theory yields the (nondispersive) homogenized wave equation, identical to (1.1) but with $a^{\varepsilon}$ replaced by its $G$-limit $a^{0}$, which no longer exhibits any small-scale behavior [13]. In practice, however, it is hardly available except in a few rather special situations.

\footnotetext{
*Received by the editors October 18, 2013; accepted for publication (in revised form) April 30, 2014; published electronically September 9, 2014. This work was supported in part by the Swiss National Science Foundation.

http://www.siam.org/journals/mms/12-3/94195.html

†Mathematics Section, École Polytechnique Fédérale de Lausanne, CH-1015 Lausanne, Switzerland (assyr.abdulle@epfl.ch).

${ }^{\ddagger}$ Institute of Mathematics, University of Basel, CH-4051 Basel, Switzerland (marcus.grote@ unibas.ch).

$\S$ Institute of Mathematics, University of Basel, CH-4051 Basel, Switzerland. Current address: Laboratoire POEMS, UMA, ENSTA ParisTech, 828, Boulevard des Maréchaux, 91762 Palaiseau Cedex, France (christian.stohrer@ensta-paristech.fr).
} 
In [21], a numerical method based on asymptotic expansions [12] was proposed for the homogenization of (1.1) with $a^{\varepsilon}$ uniformly periodic and with special symmetry. Alternatively, upscaling methods $[35,32,14]$ make no assumption about scale separation or the structure of $a^{\varepsilon}$ but compute an effective coarse-scale model directly from the fully resolved wave equation in the entire computational domain; hence, the initial set-up cost for the coarse (upscaled) model increases as $\varepsilon \rightarrow 0$.

In contrast, heterogeneous multiscale methods (HMMs) (see, e.g., $[22,6,7])$ compute "on the fly" an effective equation at the macroscale from local microproblems restricted to sampling domains proportional in size to $\varepsilon$; hence, the total computational cost remains independent of the microscale. Recently, Engquist, Holst, and Runborg [24] proposed an FD-HMM scheme for (1.1). A finite element heterogeneous multiscale method (FE-HMM) was later proposed in [8] and shown to yield optimal convergence to the limit, $u^{0}$, from classical homogenization theory at finite time and for a locally periodic medium.

For limited time the propagation of waves in a strongly heterogeneous medium is well described by the classical homogenized wave equation. With increasing time, however, the true solution, $u^{\varepsilon}$, deviates from the classical homogenization limit, $u^{0}$, as dispersive effects develop. To understand these dispersive effects at later times $T=\mathcal{O}\left(1 / \varepsilon^{2}\right)$, Santosa and Symes [34] derived a higher order effective equation by using Bloch waves. In one space dimension and for periodic $a^{\varepsilon}$, their derivation yields an explicit expression in the form of an effective Boussinesq-type equation:

$$
\partial_{t t} u^{\mathrm{eff}}-a^{0} \partial_{x x} u^{\mathrm{eff}}-\varepsilon^{2} b^{0} \partial_{x x x x} u^{\mathrm{eff}}=F .
$$

Here $a^{0}$ corresponds to the effective coefficient from classical homogenization theory, whereas $b^{0}>0$, which was later rederived by formal asymptotic expansion in [15]. In [25], the FD-HMM from [24] was enhanced to capture those long-time dispersive effects, but it now requires increasingly larger space-time sampling domains as $\varepsilon \rightarrow 0$, together with high-order macro-micro coupling and correction to the initial data. Moreover, since the FD-HMM solution converges with decreasing mesh size to the solution of (1.2), which is ill-posed, regularization is also needed.

In [30], Lamacz rigorously proved that $u^{\varepsilon}$ can be approximated with error $\mathcal{O}(\varepsilon)$ (in an $L^{\infty}$-norm) up to time $T=\mathcal{O}\left(1 / \varepsilon^{2}\right)$ by the solution $u^{\text {eff }}$ of the well-posed onedimensional limit equation

$$
\partial_{t t} u^{\mathrm{eff}}-a^{0} \partial_{x x} u^{\mathrm{eff}}-\varepsilon^{2} \frac{b^{0}}{a^{0}} \partial_{t t} \partial_{x x} u^{\mathrm{eff}}=F .
$$

Even for one-dimensional problems, however, the coefficient $b^{0}>0$ relies on a "cascade" of cell problems and is therefore hardly straightforward to calculate. Note that (1.3) coincides with (1.2) if time derivatives are formally replaced by space derivatives in the third term. By using Bloch-wave techniques, the analysis from [30] was recently extended to higher dimensions [20].

The weak formulation of (1.3) suggests that an effective correction at the macroscale is also needed in the $L^{2}$ inner product term that involves $\partial_{t t} u^{\text {eff }}$. This insight led in [9] to a new FE heterogeneous multiscale method for long times, which we named FE-HMM-L. In contrast to the FD-HMM from [24], the FE-HMM-L relies on timeindependent cell problems, approximates a well-posed effective equation, and requires no particular high-order numerical approximation at the macro-level. Moreover, the FE-HMM-L adds no computational cost and makes the same assumptions about the structure of $a^{\varepsilon}$ as the FE-HMM, that is, stationarity and scale separation, which underlie all HMM schemes. 
The remainder of this paper is organized as follows. In section 2, we first recall some known analytical results from homogenization theory. Next, in section 3, we present the FE-HMM-L method for the wave equation (1.1). In section 4, we first establish that the FE-HMM-L method is well defined regardless of $\varepsilon$ or the mesh size. Then, we state optimal a priori error bounds with respect to the energy norm and the $L^{2}$-norm for finite time $T>0$, which are proved subsequently. As a consequence, the FE-HMM-L approach is consistent with classical numerical homogenization on any fixed time interval $[0, T]$. Finally in section 5 , we present a series of numerical experiments in one and two space dimensions that corroborate the expected optimal convergence rates of the FE-HMM-L and demonstrate its ability to capture the longtime dispersive effects on much longer time intervals $\left[0, T / \varepsilon^{2}\right]$.

1.1. Notation. Let $\Omega \subset \mathbb{R}^{d}$ be open and denote by $W^{k, p}(\Omega)$ the standard Sobolev space. For $p=2$, we also use $H^{k}(\Omega)$ and $H_{0}^{1}(\Omega)$, and we denote by $H_{\mathrm{per}}^{k}(Y)$ the closure of $C_{\text {per }}^{\infty}(Y)$ (the subset of $C^{\infty}\left(\mathbb{R}^{d}\right)$ of periodic functions in the unit cube $\left.Y=(-1 / 2,1 / 2)^{d}\right)$ with respect to the $H^{k}$-norm. Next, we let $W_{\mathrm{per}}^{1}(Y)=\{v \in$ $\left.H_{\text {per }}^{1}(Y) ; \int_{Y} v d x=0\right\}$ and denote by $|D|$ the measure of a set $D \subset \Omega$. For $T>0$ and $B$ a Banach space with norm $\|\cdot\|_{B}$, we denote by $L^{p}(0, T ; B)=L^{p}(B), 1 \leq p \leq \infty$, the Bochner space of functions $v:(0, T) \rightarrow B$. Equipped with the norm

$$
\|v\|_{L^{p}(0, T ; B)}= \begin{cases}\left(\int_{0}^{T}\|v(t)\|_{B}^{p} d t\right)^{\frac{1}{p}} & \text { for } p<\infty, \\ \operatorname{ess} \sup \|v(t)\|_{B} & \text { for } p=\infty,\end{cases}
$$

the space $L^{p}(0, T ; B)$ is also a Banach space [27].

2. Model problem. We let $\Omega$ be a convex polyhedron in $\mathbb{R}^{d}, 1 \leq d \leq 3$, and consider the following variational formulation of the wave equation (1.1):

Find $u^{\varepsilon}:[0, T] \rightarrow H_{0}^{1}(\Omega)$ such that

$$
\begin{cases}\left(\partial_{t t} u^{\varepsilon}(t), v\right)+B^{\varepsilon}\left(u^{\varepsilon}(t), v\right)=(F(t), v) & \forall v \in H_{0}^{1}(\Omega), \\ u^{\varepsilon}(0)=f, \quad \partial_{t} u^{\varepsilon}(0)=g & \text { in } \Omega,\end{cases}
$$

where $(\cdot, \cdot)$ denotes the standard $L^{2}$ inner product over $\Omega$ and the bilinear form $B^{\varepsilon}$ is given by

$$
B^{\varepsilon}(v, w)=\int_{\Omega} a^{\varepsilon}(x) \nabla v(x) \cdot \nabla w(x) d x \quad \forall v, w \in H_{0}^{1}(\Omega) .
$$

We also assume that $a^{\varepsilon} \in L^{\infty}\left(\Omega ; \mathbb{R}^{d \times d}\right)$ is symmetric, uniformly elliptic, and bounded; i.e., there exist $0<\lambda \leq \Lambda$ such that for all $\xi \in \mathbb{R}^{d}$ and for all $\varepsilon>0$

$$
\lambda|\xi|^{2} \leq a^{\varepsilon}(x) \xi \cdot \xi \leq \Lambda|\xi|^{2} \quad \text { a.e. } x \in \Omega .
$$

Hence the bilinear form $B^{\varepsilon}$ is symmetric, uniformly elliptic, and bounded on $H_{0}^{1}(\Omega)$. Furthermore, we make the following standard regularity assumptions:

$$
F \in L^{2}\left(0, T ; L^{2}(\Omega)\right), \quad f \in H_{0}^{1}(\Omega), \quad g \in L^{2}(\Omega) .
$$

In (2.1), we have imposed homogeneous Dirichlet conditions for simplicity, but clearly other boundary conditions could be used. 
Under assumptions (2.3), (2.4), the wave equation (2.1) has a unique (weak) solution $u^{\varepsilon} \in L^{2}\left(0, T ; H_{0}^{1}(\Omega)\right)$ with $\partial_{t} u^{\varepsilon} \in L^{2}\left(0, T ; L^{2}(\Omega)\right)$. In fact, the solution is more regular, as $u^{\varepsilon} \in L^{\infty}\left(0, T ; H_{0}^{1}(\Omega)\right)$ with $\partial_{t} u^{\varepsilon} \in L^{\infty}\left(0, T ; L^{2}(\Omega)\right)$. We even have

$$
u^{\varepsilon} \in C\left([0, T] ; H_{0}^{1}(\Omega)\right), \quad \partial_{t} u^{\varepsilon} \in C\left([0, T] ; L^{2}(\Omega)\right)
$$

after redefinition on a set of measure zero [31].

2.1. Homogenization theory. Following the macro- to microscale HMM approach $[3,4,7]$, we must first identify an appropriate macroscale model. For limited time the propagation of waves in a rapidly varying medium is well described by the (nondispersive) homogenized wave equation whose variational formulation reads as follows:

Find $u^{0}:[0, T] \rightarrow H_{0}^{1}(\Omega)$ such that

$$
\begin{cases}\left(\partial_{t t} u^{0}(t), v\right)+B^{0}\left(u^{0}(t), v\right)=F & \forall v \in H_{0}^{1}(\Omega), \\ u^{0}(0)=f, \quad \partial_{t} u^{0}(0)=g & \text { in } \Omega,\end{cases}
$$

where

$$
B^{0}(v, w)=\int_{\Omega} a^{0}(x) \nabla v(x) \cdot \nabla w(x) d x \quad \forall v, w \in H_{0}^{1}(\Omega) .
$$

Note that $a^{0}$, the $G$-limit of $a^{\varepsilon}$, no longer exhibits any microscopic behavior and also satisfies $(2.3)$; see $[18,12]$ for details. Hence, on a fixed time interval $[0, T]$, the true solution $u^{\varepsilon}$ of (1.1) indeed converges in a weak sense to $u^{0}$, the solution of the homogenized wave equation (2.5).

With increasing time, however, $u^{\varepsilon}$ deviates from the classical homogenization limit, as a large secondary wave train develops unexpectedly because of a subtle interplay between smaller scales. To capture this dispersive behavior, Lamacz [30] proposed the effective Boussinesq-type equation (1.3) and proved that its solution approximates $u^{\varepsilon}$ with error $\mathcal{O}(\varepsilon)$ (in an $L^{\infty}$-norm), even on increasingly longer time intervals $\left[0, T / \varepsilon^{2}\right]$.

Now, multiplication of (1.3) with a test function and integration by parts motivates the following effective macroscale model:

Find $u^{\text {eff }}:[0, T] \rightarrow H_{0}^{1}(\Omega)$ such that

$$
\left\{\begin{array}{cl}
\left(\partial_{t t} u^{\mathrm{eff}}(t), v\right)^{\mathrm{eff}}+B^{0}\left(u^{\varepsilon}(t), v\right)=0 & \forall v \in H_{0}^{1}(\Omega), \\
u^{\varepsilon}(0)=f, \quad \partial_{t} u^{\varepsilon}(0)=g & \text { in } \Omega,
\end{array}\right.
$$

where the effective inner product $(\cdot, \cdot)^{\text {eff }}$ may depend on the spatial derivative of its arguments. Note that we recover the classical homogenized wave equation by replacing the effective inner product with the standard $L^{2}$-product. On the other hand, if we let

$$
(v, w)^{\mathrm{eff}}=(v, w)+\varepsilon^{2}\left(\frac{b^{0}}{a^{0}} \nabla v, \nabla w\right),
$$

we recover the variational formulation of the dispersive effective equation (1.3). 
3. Multiscale FEM for the wave equation. In this section, we propose a new finite element heterogeneous multiscale method for long-time wave propagation (FE-HMM-L), which is based on the macroscale model (2.7). In (2.7), neither the effective bilinear form nor the effective inner product is available, as $a^{0}$ and $b^{0}$ are not explicitly known a priori. Instead, the FE-HMM-L method recovers the required information locally by solving "on-the-fly" appropriate microscale problems which yield the following:

1. A modified bilinear form $B_{H}$ based on microfunctions defined on sampling domains;

2. A modified inner product $(\cdot, \cdot)_{Q}=(\cdot, \cdot)_{H}+(\cdot, \cdot)_{M}$, where $\left(v_{H}, w_{H}\right)_{H}$ denotes the standard $L^{2}$ inner product with numerical quadrature, whereas the additional inner product $\left(v_{H}, w_{H}\right)_{M}$ (defined below) utilizes the same microfunctions as $B_{H}$.

Both the modified bilinear form and the two inner products are based on numerical quadrature. Hence, let $\left(\hat{x}_{j}, \hat{\omega}_{j}\right)$ for $j=1, \ldots, J$, respectively, denote the quadrature nodes and weights of a quadrature formula $(\mathrm{QF})$ for the reference element $\hat{K}$. We assume that

$$
\hat{\omega}_{j}>0, \quad j=1, \ldots, J,
$$

and that there exists a $\hat{\lambda}>0$ such that

$$
\begin{aligned}
\sum_{j=1}^{J} \hat{\omega}_{j}\left|\nabla \hat{p}\left(\hat{x}_{j}\right)\right|^{2} & \geq \hat{\lambda}\|\nabla \hat{p}\|_{L^{2}(\hat{K})}^{2} & & \forall \hat{p} \in \mathcal{R}^{\ell}(\hat{K}), \\
\int_{\hat{K}} \hat{p}(\hat{x}) d \hat{x} & =\sum_{j=1}^{J} \hat{\omega}_{j} \hat{p}\left(\hat{x}_{j}\right) & & \forall \hat{p} \in \mathcal{R}^{\sigma}(\hat{K}) .
\end{aligned}
$$

Here $\sigma=\max (2 \ell-2, \ell)$ and $\mathcal{R}^{\sigma}(\hat{K})$ is the space $\mathcal{P}^{\sigma}(\hat{K})$ of polynomials on $\hat{K}$ of total degree at most $\sigma$ if $\hat{K}$ is a simplicial element, or $\sigma=\max (2 \ell-1, \ell+1)$ and $\mathcal{R}^{\sigma}(\hat{K})$ is the space $\mathcal{Q}^{\sigma}(\hat{K})$ of polynomials on $\hat{K}$ of degree at most $\sigma$ in each variable if $\hat{K}$ is a quadrilateral element. Moreover, for the QF used in $(\cdot, \cdot)_{H}$, we assume that

$$
\sum_{j=1}^{J} \hat{\omega}_{j}\left|\hat{p}\left(\hat{x}_{j}\right)\right|^{2} \geq \hat{\lambda}\|\hat{p}\|_{L^{2}(\hat{K})}^{2} \quad \forall \hat{p} \in \mathcal{R}^{\ell}(\hat{K}) .
$$

Remark 3.1. Assumptions (3.1)-(3.3) are standard for retaining optimal convergence rates of FEMs with numerical quadrature [16]. In fact, for time-dependent problems, (3.4) must hold for the QF used in the assembly of the mass-matrix; see [33] for parabolic and [11] for hyperbolic problems. Note that (3.4) implies (3.2).

3.1. Macro- and micro-FE spaces. We consider a shape-regular macroscopic triangulation, $\mathcal{T}_{H}$, of $\Omega$ into simplicial or quadrilateral elements $K$ of maximal diameter $H$; for simplicity, we assume that $\Omega$ is a polygon. By macroscopic we mean that $\mathcal{T}_{H}$ does not have to resolve the microstructure of the medium, i.e., $H \gg \varepsilon$ is allowed. On $\mathcal{T}_{H}$ we define the standard FE space

$$
S_{0}^{\ell}\left(\Omega, \mathcal{T}_{H}\right)=\left\{v_{H} \in H_{0}^{1}(\Omega) ;\left.v_{H}\right|_{K} \in \mathcal{R}^{\ell}(K) \forall K \in \mathcal{T}_{H}\right\} .
$$

Every element $K$ in $\mathcal{T}_{H}$ is assumed affine equivalent to the reference element, $\hat{K}$, and we denote the associated affine mapping by $F_{K}: \hat{K} \rightarrow K$. 
For the microproblems we consider inside each $K$ sampling domains $K_{\delta}$ of size $\delta$, centered about suitable quadrature points. In general, $\delta \geq \varepsilon$ is comparable in size to $\varepsilon$, yet for locally periodic problems we usually set $\delta=\varepsilon$. On each sampling domain, we then consider a (micro-)partition $\mathcal{T}_{h}$ of $K_{\delta}$ into simplicial or quadrilateral elements $Q \in \mathcal{T}_{h}$ and a micro-FE space of periodic functions

$$
S^{q}\left(K_{\delta}, \mathcal{T}_{h}\right)=\left\{v_{h} \in W\left(K_{\delta}\right) ;\left.v_{h}\right|_{Q} \in \mathcal{R}^{q}(Q) \forall Q \in \mathcal{T}_{h}\right\},
$$

where for a periodic coupling

$$
W\left(K_{\delta}\right)=W_{\mathrm{per}}^{1}\left(K_{\delta}\right)=\left\{v \in H_{\mathrm{per}}^{1}\left(K_{\delta}\right) ; \int_{K_{\delta}} v d x=0\right\},
$$

and for a coupling through Dirichlet boundary conditions

$$
W\left(K_{\delta}\right)=H_{0}^{1}\left(K_{\delta}\right)
$$

3.2. The FE-HMM-L method. To define the FE-HMM-L method, we first choose on each element $K \in \mathcal{T}_{H}$ two QFs, $\left(x_{K, j}, \omega_{K, j}\right)$ for $j=1, \ldots, J$ and $\left(x_{K, j}^{\prime}, \omega_{K, j}^{\prime}\right)$ for $j=1, \ldots, J^{\prime}$, both usually determined through the affine mapping $x=F_{K}(\hat{x})$. To each quadrature node $x_{K, j}$ we associate a sampling domain $K_{\delta}$, centered about $x_{K, j}$,

$$
K_{\delta}=K_{\delta}\left(x_{K, j}\right)=x_{K, j}+\delta Y, \quad Y=(-1 / 2,1 / 2)^{d},
$$

together with the linearization $v_{H, \text { lin }}(x)$ of any function $v_{H} \in S_{0}^{\ell}\left(\Omega, \mathcal{T}_{H}\right)$,

$$
v_{H, \operatorname{lin}}(x)=v_{H}\left(x_{K, j}\right)+\left(x-x_{K, j}\right) \cdot \nabla v_{H}\left(x_{K, j}\right) .
$$

Then, the FE-HMM-L method is defined as follows:

Find $u_{H}:[0, T] \rightarrow S_{0}^{\ell}\left(\Omega, \mathcal{T}_{H}\right)$ such that

$$
\begin{cases}\left(\partial_{t t} u_{H}(t), v_{H}\right)_{Q}+B_{H}\left(u_{H}(t), v_{H}\right)=\left(F(t), v_{H}\right) & \forall v_{H} \in S_{0}^{\ell}\left(\Omega, \mathcal{T}_{H}\right), \\ u_{H}(0)=f_{H}, \quad \partial_{t} u_{H}(0)=g_{H} & \text { in } \Omega,\end{cases}
$$

where $f_{H}, g_{H} \in S_{0}^{\ell}\left(\Omega, \mathcal{T}_{H}\right)$ are suitable approximations of the initial conditions, and

$$
\begin{aligned}
& B_{H}\left(v_{H}, w_{H}\right)=\sum_{K \in \mathcal{T}_{H}} \sum_{j=1}^{J} \frac{\omega_{K, j}}{\left|K_{\delta}\right|} \int_{K_{\delta}} a^{\varepsilon}(x) \nabla v_{h}(x) \cdot \nabla w_{h}(x) d x \\
& \left(v_{H}, w_{H}\right)_{Q}=\left(v_{H}, w_{H}\right)_{H}+\left(v_{H}, w_{H}\right)_{M}, \\
& \left(v_{H}, w_{H}\right)_{H}=\sum_{K \in \mathcal{T}_{H}} \sum_{j=1}^{J^{\prime}} \omega_{K, j}^{\prime} v_{H}\left(x_{K, j}^{\prime}\right) w_{H}\left(x_{K, j}^{\prime}\right), \\
& \left(v_{H}, w_{H}\right)_{M}=\sum_{K \in \mathcal{T}_{H}} \sum_{j=1}^{J} \frac{\omega_{K, j}}{\left|K_{\delta}\right|} \int_{K_{\delta}}\left(v_{h}(x)-v_{H, \operatorname{lin}}(x)\right)\left(w_{h}(x)-v_{H, \operatorname{lin}}(x)\right) d x .
\end{aligned}
$$

Both $B_{H}(\cdot, \cdot)$ and $(\cdot, \cdot)_{M}$ involve microfunctions $v_{h}$ (resp., $w_{h}$ ) that are given by the following:

Find $v_{h}$, with $\left(v_{h}-v_{H, \text { lin }}\right) \in S^{q}\left(K_{\delta}, \mathcal{T}_{h}\right)$, such that

$$
\int_{K_{\delta}} a^{\varepsilon}(x) \nabla v_{h} \cdot \nabla z_{h} d x=0 \quad \forall z_{h} \in S^{q}\left(K_{\delta}, \mathcal{T}_{h}\right) .
$$

Copyright (c) by SIAM. Unauthorized reproduction of this article is prohibited. 
Because of (2.3) and the Lax-Milgram theorem, every microproblem (3.16) has a unique solution. The microfunctions $v_{h}$ (resp., $w_{h}$ ) depend on the corresponding macrofunctions $v_{H}$ (resp., $w_{H}$ ) through the periodic coupling across the boundaries of the sampling domains in (3.16); note that $v_{H \text {,lin }}$, defined in (3.10), also depends on $x_{K, j}$.

Following [7], we shall now reformulate the bilinear form $B_{H}$ directly in terms of the macrofunctions $v_{H}, w_{H}$. To do so, we first write $v_{h}$ as

$$
v_{h}(x)=v_{H, \operatorname{lin}}(x)+\psi_{h}(x) \cdot \nabla v_{H}\left(x_{K, j}\right),
$$

where each component $\psi_{h}^{i} \in S^{q}\left(K_{\delta}, \mathcal{T}_{h}\right)$ of $\psi_{h}(x)=\left(\psi_{h}^{1}(x), \psi_{h}^{2}(x), \ldots, \psi_{h}^{d}(x)\right)^{T}$ solves

$$
\int_{K_{\delta}} a^{\varepsilon}(x) \nabla \psi_{h}^{i} \cdot \nabla z_{h} d x=-\int_{K_{\delta}} a^{\varepsilon}(x) e_{i} \cdot \nabla z_{h} d x \quad \forall z_{h} \in S^{q}\left(K_{\delta}, \mathcal{T}_{h}\right),
$$

with $e_{i}$ the $i$ th canonical basis vector of $\mathbb{R}^{d}$. By using (3.17) in (3.12), we now reformulate $B_{H}$ as

$$
B_{H}\left(v_{H}, w_{H}\right)=\sum_{K \in \mathcal{T}_{H}} \sum_{j=1}^{J} \omega_{K, j} a_{K}^{0}\left(x_{K, j}\right) \nabla v_{H}\left(x_{K, j}\right) \cdot \nabla w_{H}\left(x_{K, j}\right) .
$$

Here $a_{K}^{0}\left(x_{K, j}\right)$ is defined by

$$
a_{K}^{0}\left(x_{K, j}\right)=\frac{1}{\left|K_{\delta}\left(x_{K, j}\right)\right|} \int_{K_{\delta}\left(x_{K, j}\right)} a^{\varepsilon}(x)\left(I+J_{\psi_{h}}^{T}(x)\right) d x,
$$

where $J_{\psi_{h}}^{T}(x)$ corresponds to the $d \times d$ matrix

$$
\left(J_{\psi_{h}}(x)\right)_{r s}=\frac{\partial}{\partial x_{s}} \psi_{h}^{r}(x)
$$

with $\psi_{h}^{i}$ given by (3.18). We also define

$$
\bar{a}_{K}^{0}\left(x_{K, j}\right)=\frac{1}{\left|K_{\delta_{j}}\right|} \int_{K_{\delta_{j}}} a^{\varepsilon}(x)\left(I+J_{\psi}^{T}(x)\right) d x,
$$

where $J_{\psi(x)}$ is defined similarly as $J_{\psi_{h}(x)}$, but with $\psi_{h}^{i}$ replaced by $\psi^{i}$, the solutions of the continuous counterpart of (3.18) set in the Sobolev space $W\left(K_{\delta}\right)$ (see (3.7), $(3.8))$.

Remark 3.2. In (3.12), (3.13), the inner product $(\cdot, \cdot)_{H}$ corresponds to the standard approximation of the $L^{2}$ inner product with the $\mathrm{QF}\left\{x_{K, j}^{\prime}, \omega_{K, j}^{\prime}\right\}$, whereas $B_{H}$ corresponds to the standard FE-HMM bilinear form; see $[7,3]$ for a review. Since the modified inner product in (3.13) relies on the same microfunctions as $B_{H}$, no additional microproblems need to be solved. Hence by choosing the same QF for $B_{H}$ and $(\cdot, \cdot)_{M}$, we keep the computational cost identical to that of the FE-HMM method from [8], where no effective inner product was used. In fact, the analysis below would remain valid with the inclusion of a third $\mathrm{QF}$, or even numerical integration for the source term, though without any added insight.

Copyright $@$ by SIAM. Unauthorized reproduction of this article is prohibited. 
4. Error estimates. We now establish the well-posedness of the FE-HMM-L method from section 3.2, regardless of $\varepsilon$ or the mesh size. Then, we state optimal a priori error bounds with respect to the energy norm and the $L^{2}$-norm for finite time $T>0$, which are proved subsequently. Hence, on $[0, T]$, the FE-HMM-L approach is indeed consistent with classical numerical homogenization. We remark that in the definition (3.11) of the FE-HMM-L method, we have not yet specified how to approximate the initial conditions. Here we shall use standard nodal interpolation, $f_{H}=I_{H} f$ and $g_{H}=I_{H} g$, but wish to emphasize that other approximations are possible.

4.1. Preliminaries. Here we assume that the homogenized tensor $a^{0}$ is sufficiently regular. In particular, for $a^{0} \in\left(W^{1, \infty}(\Omega)\right)^{d \times d}$ we consider the bilinear form

$$
B_{H}^{0}\left(v_{H}, w_{H}\right)=\sum_{K \in \mathcal{T}_{H}} \sum_{j=1}^{J} \omega_{K, j} a^{0}\left(x_{K, j}\right) \nabla v_{H}\left(x_{K, j}\right) \cdot \nabla w_{H}\left(x_{K, j}\right),
$$

which results from applying a standard FEM with numerical quadrature to (2.5). For $m \geq 1$, the following broken norms will sometimes be used for piecewise smooth functions:

$$
\|v\|_{\bar{H}^{m}(\Omega)}:=\left(\sum_{K \in \mathcal{T}_{H}}\|v\|_{H^{m}}^{2}\right)^{1 / 2} .
$$

Then, provided that there is sufficient regularity of $a^{0}$ and that assumptions (3.1)(3.4) hold, we have the following estimates for $v_{H}, w_{H} \in S^{\ell}\left(\Omega, \mathcal{T}_{H}\right)$ and $\mu=0,1$ :

$$
\begin{aligned}
&\left|B^{0}\left(v_{H}, w_{H}\right)-B_{H}^{0}\left(v_{H}, w_{H}\right)\right| \leq C H^{\ell+\mu} \max _{i, j}\left\|a_{i j}^{0}\right\|_{W^{\ell+\mu, \infty}(\Omega)} \\
& \cdot\left\|v_{H}\right\|_{\bar{H}^{\ell+\mu}(\Omega)}\left\|w_{H}\right\|_{\bar{H}^{1+\mu}(\Omega)} \\
&\left|B^{0}\left(v_{H}, w_{H}\right)-B_{H}^{0}\left(v_{H}, w_{H}\right)\right| \leq C H \max _{i, j}\left\|a_{i j}^{0}\right\|_{W^{1, \infty}(\Omega)}\left\|v_{H}\right\|_{H^{1}(\Omega)}\left\|w_{H}\right\|_{H^{1}(\Omega)}, \\
& B_{H}^{0}\left(v_{H}, v_{H}\right) \geq c\left\|v_{H}\right\|_{H^{1}(\Omega)}^{2} \\
&\left|\left(v_{H}, w_{H}\right)-\left(v_{H}, w_{H}\right)_{H}\right| \leq C H^{\ell+\mu}\left\|v_{H}\right\|_{\bar{H}^{\ell+\mu}(\Omega)}\left\|w_{H}\right\|_{\bar{H}^{1+\mu}(\Omega)}
\end{aligned}
$$

and, for $\left\|v_{H}\right\|_{H}^{2}=\left(v_{H}, v_{H}\right)_{H}$,

$$
c_{1}\left\|v_{H}\right\|_{L^{2}(\Omega)} \leq\left\|v_{H}\right\|_{H} \leq c_{2}\left\|v_{H}\right\|_{L^{2}(\Omega)} .
$$

Here the constants $C, c, c_{1}, c_{2}>0$ are all independent of $H$. Note that only assumptions (3.1)-(3.3) are needed for (4.2)-(4.4), whereas to prove (4.6), the stricter assumption (3.4) for $\left(x_{K, j}^{\prime}, \omega_{K, j}^{\prime}\right)$ is necessary; see $[16,17]$ for details.

We also let $I_{H}$ denote an interpolation operator, such as the standard nodal interpolant (see [16, Chap. 3.2]), which satisfies, for all integers $m, k$ with $0 \leq m \leq 1$ and $2 \leq k \leq \ell+1$,

$$
\left\|v-I_{H} v\right\|_{H^{m}(\Omega)} \leq C H^{k-m}\|v\|_{H^{k}(\Omega)}
$$

for all $v \in H^{k}(\Omega) \cap H_{0}^{1}(\Omega)$ and $I_{H} v \in S_{0}^{\ell}\left(\Omega, \mathcal{T}_{H}\right)$. Clearly, other interpolants which might require less regularity could also be used. 
The following lemma has been proved in various forms $[3,5,23]$.

Lemma 4.1. Suppose that (3.1)-(3.3) hold. Then, for all $v_{H}, w_{H} \in S^{\ell}\left(\Omega, \mathcal{T}_{H}\right)$, we have

$$
\left|B_{H}^{0}\left(v_{H}, w_{H}\right)-B_{H}\left(v_{H}, w_{H}\right)\right| \leq e_{H M M}\left\|\nabla v_{H}\right\|_{L^{2}(\Omega)}\left\|\nabla w_{H}\right\|_{L^{2}(\Omega)},
$$

where

$$
e_{H M M}=\sup _{\substack{K \in \mathcal{T}_{H} \\ 1 \leq j \leq J}}\left\|a^{0}\left(x_{K, j}\right)-a_{K}^{0}\left(x_{K, j}\right)\right\|_{F}
$$

and $\|\cdot\|_{F}$ denotes the Frobenius norm.

Moreover, for all $v_{H}, w_{H} \in S^{\ell}\left(\Omega, \mathcal{T}_{H}\right)$, we have

$$
B_{H}\left(v_{H}, v_{H}\right) \geq \gamma\left\|v_{H}\right\|_{H^{1}(\Omega)}^{2}
$$

and

$$
\left|B_{H}\left(v_{H}, w_{H}\right)\right| \leq \Gamma\left\|v_{H}\right\|_{H^{1}(\Omega)}\left\|w_{H}\right\|_{H^{1}(\Omega)},
$$

where $\gamma, \Gamma>0$.

Next, we decompose the combined modeling error and microerror, $e_{\mathrm{HMM}}$, in (4.8) as

$$
\left\|a^{0}\left(x_{K, j}\right)-a_{K}^{0}\left(x_{K, j}\right)\right\|_{F} \leq \underbrace{\left\|a^{0}\left(x_{K, j}\right)-\bar{a}_{K}^{0}\left(x_{K, j}\right)\right\|_{F}}_{\text {err } r_{\text {mod }}}+\underbrace{\left\|\bar{a}_{K}^{0}\left(x_{K, j}\right)-a_{K}^{0}\left(x_{K, j}\right)\right\|_{F}}_{\text {err } \text { mic }} .
$$

Here $a^{0}\left(x_{K, j}\right)$ corresponds to the homogenized tensor evaluated at the quadrature point $x_{K, j}$, whereas the tensors $\bar{a}^{0}\left(x_{K, j}\right)$ and $a_{K}^{0}\left(x_{K, j}\right)$ (numerical approximations of $\left.a^{0}\left(x_{K, j}\right)\right)$ are defined in (3.20) and (3.19), respectively.

The microerror, $\operatorname{err}_{m i c}$, describes the error due to the micro-FEM and can be analyzed without any assumption about spatial structure (e.g., periodicity or random stationarity). For piecewise linear microfunctions $[1,2]$ or for higher-order piecewise polynomial microfunctions [5, Lemma 5.2], the following result holds.

If the solutions $\psi^{i}$ of the continuous counterpart of (3.18) in the Sobolev space $W\left(K_{\delta_{j}}\right)$ satisfy $\psi^{i} \in H^{q+1}\left(K_{\delta}\right)$ and

$$
\left|\psi^{i}\right|_{H^{q+1}\left(K_{\delta}\right)} \leq C \varepsilon^{-q} \sqrt{\left|K_{\delta}\right|}, \quad K \in \mathcal{T}_{H}, \quad i=1, \ldots, d,
$$

then we have for any $K \in \mathcal{T}_{H}$ the estimate

$$
e r r_{m i c}=\left\|\bar{a}_{K}^{0}-a_{K}^{0}\right\|_{F} \leq C\left(\frac{h}{\varepsilon}\right)^{2 q} .
$$

The modeling error, $e r r_{m o d}$, quantifies how well the upscaling procedure captures the effective homogenized coefficient $a^{0}$. To estimate it, an assumption about spatial structure, such as local periodicity or random stationarity, is needed. For instance, in the case of locally periodic data, i.e., where $a^{\varepsilon}(x)=a\left(x, \frac{x}{\varepsilon}\right)=a(x, y)$ is periodic in $y$ and $a_{i j}(x, y) \in W^{1, \infty}\left(\bar{\Omega}, L^{\infty}(Y)\right), 1 \leq i, j \leq d$ (see $[23,10]$ ), the modeling error can be estimated as

$$
e r r_{\text {mod }}=\left\|a^{0}\left(x_{K}\right)-\bar{a}_{K}^{0}\right\|_{F} \leq \begin{cases}0 & \text { if } W\left(K_{\delta}\right)=W_{p e r}^{1}\left(K_{\delta}\right) \text { and } \frac{\delta}{\varepsilon} \in \mathbb{N} \\ C \frac{\varepsilon}{\delta} & \text { if } W\left(K_{\delta}\right)=H_{0}^{1}\left(K_{\delta}\right) \text { and } \delta>\varepsilon .\end{cases}
$$

Copyright (c) by SIAM. Unauthorized reproduction of this article is prohibited. 
Here we have also assumed that $a^{\varepsilon}$ is collocated in the slow variable, that is, $a^{\varepsilon}(x)=$ $a\left(x_{K}, x / \varepsilon\right)$; without this assumption, an additional term of size $\delta$ typically appears in both estimates. The modeling error has also been analyzed for random stationary tensors [23, Appendix A].

Next we derive a key identity for the $(\cdot, \cdot)_{M}$ correction to the $L^{2}$ inner product which, in particular, implies that $(\cdot, \cdot)_{Q}$ defined in (3.13) itself is a true inner product. To do so, we consider the solutions $\hat{\psi}_{h}^{i} \in S^{q}\left(Y, \hat{\mathcal{T}}_{h}\right), i=1, \ldots, d$, of the cell problems

$$
\int_{Y} a_{x_{K, j}}(y) \nabla \hat{\psi}_{h}^{i}(y) \cdot \nabla \hat{z}_{h} d y=-\int_{Y} a_{x_{K, j}}(y) e_{i} \cdot \nabla \hat{z}_{h} d y \quad \forall \hat{z}_{h} \in S^{q}\left(Y, \hat{\mathcal{T}}_{h}\right) .
$$

Here $a_{x_{K, j}}(y)=a^{\varepsilon}\left(x_{K, j}+\delta y\right)$, and $\hat{\mathcal{T}}_{h}$ denotes the mesh on the reference cell $Y$ obtained from the affine mapping $F_{K_{\delta}}: Y \rightarrow K_{\delta}$; see (3.9). Then the following lemma holds true [9].

Lemma 4.2. For all $v_{H}, w_{H} \in S^{\ell}\left(\Omega, \mathcal{T}_{H}\right)$ we have

$$
\left(v_{H}, w_{H}\right)_{M}=\varepsilon^{2} \sum_{K \in \mathcal{T}_{H}} \sum_{j=1}^{J} \omega_{K, j} M\left(x_{K, j}\right) \nabla v_{H}\left(x_{K, j}\right) \cdot \nabla w_{H}\left(x_{K, j}\right),
$$

where the symmetric $d \times d$ matrix $M\left(x_{K, j}\right)$ is defined by

$$
M_{r s}\left(x_{K, j}\right)=\left(\frac{\delta}{\varepsilon}\right)^{2} \int_{Y} \hat{\psi}_{h}^{r}(y) \hat{\psi}_{h}^{s}(y) d y .
$$

Moreover, for all $v_{H}, w_{H} \in S^{\ell}\left(\Omega, \mathcal{T}_{H}\right)$, we have

$$
\left(v_{H}, v_{H}\right)_{M} \geq 0
$$

and if $\delta=\kappa_{0} \varepsilon$, with $\kappa_{0}$ independent of $\varepsilon$,

$$
\left|\left(v_{H}, w_{H}\right)_{M}\right| \leq C \varepsilon^{2}\left\|\nabla v_{H}\right\|_{L^{2}(\Omega)}\left\|\nabla w_{H}\right\|_{L^{2}(\Omega)},
$$

where $C$ is independent of $H, h, \varepsilon, \delta$.

From Lemma 4.2 we infer that

$$
\left(v_{H}, w_{H}\right)_{Q}=\left(v_{H}, w_{H}\right)_{H}+\varepsilon^{2}\left(M v_{H}, w_{H}\right)_{H},
$$

when both QFs $\left(x_{K, j}, \omega_{K, j}\right)$ and $\left(x_{K, j}^{\prime}, \omega_{K, j}^{\prime}\right)$ are identical. Hence $(\cdot, \cdot)_{Q}$ approximates an effective inner product with numerical quadrature. It is also closely related to the effective inner product $(2.8)$. Since $(\cdot, \cdot)_{Q}$ is a true $L^{2}$-product, $(3.11)$ is equivalent to a system of linear ordinary differential equations, and there exists a unique solution $u_{H}:[0, T] \rightarrow S^{\ell}\left(\Omega, \mathcal{T}_{H}\right)$ of (3.11). We summarize this result in the following corollary.

Corollary 4.3. The FE-HMM-L method (3.11)-(3.15) has a unique solution $u_{H} \in L^{\infty}\left(0, T ; S^{\ell}\left(\Omega, \mathcal{T}_{H}\right)\right)$ for all $\varepsilon, h, H>0$.

4.2. Main results. For $\varepsilon, H, h \rightarrow 0$, the FE-HMM-L solution $u_{H}$ converges to the solution $u^{0}$ of the homogenized wave equation (2.5) at finite time. In the following two theorems we state the precise error bounds which lead to optimal convergence rates with respect to the energy and the $L^{2}$-norm. Their proofs are postponed to section 4.3 .

THEOREM 4.4. Let $u^{0}$ and $u_{H}$ be the solutions of (2.5) and (3.11), respectively. Suppose that (4.2)-(4.6) hold for $\mu=0$, and also that (4.7) holds. Moreover, assume

Copyright $@$ by SIAM. Unauthorized reproduction of this article is prohibited. 
that

$$
\begin{aligned}
& \partial_{t}^{k} u^{0} \in L^{2}\left(0, T ; H^{\ell+1}(\Omega)\right), \quad \partial_{t}^{2+k} u^{0} \in L^{2}\left(0, T ; H^{\ell}(\Omega)\right), \quad k=0,1,2, \\
& a_{i, j}^{0} \in W^{\ell, \infty}(\Omega), \quad i, j=1, \ldots, d, \quad f \in H^{\ell+1}(\Omega), \quad g \in H^{\max (2, \ell)}(\Omega), \\
& \partial_{t}^{k} u_{H} \in L^{2}\left(0, T ; H^{1}(\Omega)\right), \quad 0 \leq k \leq 2, \quad\left\|u_{H}\right\|_{H^{1}\left(0, T ; H^{1}(\Omega)\right)} \leq c
\end{aligned}
$$

independently of $H$. Then,

$$
\left\|\partial_{t}\left(u^{0}-u_{H}\right)\right\|_{L^{\infty}\left(0, T ; L^{2}(\Omega)\right)}+\left\|u^{0}-u_{H}\right\|_{L^{\infty}\left(0, T ; H^{1}(\Omega)\right)} \leq C\left(H^{\ell}+e_{H M M}+\varepsilon^{2}\right)
$$

for $\varepsilon \leq H \leq H_{0}$.

THEOREM 4.5. Let $u^{0}$ and $u_{H}$ be the solutions of (2.5) and (3.11), respectively. Suppose that (4.2)-(4.6) hold for $\mu=1$, and also that (4.7) hold. Moreover, assume that

$$
\begin{aligned}
& \partial_{t}^{k} u^{0} \in L^{2}\left(0, T ; H^{\ell+1}(\Omega)\right), \quad \partial_{t}^{4} u^{0} \in L^{2}\left(0, T ; H^{\ell}(\Omega)\right), \quad k=0,1,2,3, \\
& a_{i, j}^{0} \in W^{\ell+1, \infty}(\Omega), \quad i, j=1, \ldots, d, \quad f \in H^{\ell+1}(\Omega), \\
& \partial_{t}^{k} u_{H} \in L^{2}\left(0, T ; H^{1}(\Omega)\right), \quad k=0,1, \quad\left\|u_{H}\right\|_{H^{1}\left(0, T ; L^{2}(\Omega)\right)} \leq c
\end{aligned}
$$

independently of $H$. Then,

$$
\left\|u^{0}-u_{H}\right\|_{L^{\infty}\left(0, T ; L^{2}(\Omega)\right)} \leq C\left(H^{\ell+1}+e_{H M M}+\varepsilon^{2}\right)
$$

for $\varepsilon \leq H \leq H_{0}$.

By combining the results of Theorems 4.4 and 4.5 with the error bound for $e_{\mathrm{HMM}}$ described in section 4.1, we obtain the following two fully discrete error estimates under appropriate regularity of the microsolution (see (4.11)):

$$
\begin{aligned}
& \left\|\partial_{t}\left(u^{0}-u_{H}\right)\right\|_{L^{\infty}\left(0, T ; L^{2}(\Omega)\right)}+\left\|u^{0}-u_{H}\right\|_{L^{\infty}\left(0, T ; H^{1}(\Omega)\right)} \\
& \quad \leq C\left(H^{\ell}+\left(\frac{h}{\varepsilon}\right)^{2 q}+e r r_{\text {mod }}+\varepsilon^{2}\right), \\
& \left\|u^{0}-u_{H}\right\|_{L^{\infty}\left(0, T ; L^{2}(\Omega)\right)} \leq C\left(H^{\ell+1}+\left(\frac{h}{\varepsilon}\right)^{2 q}+e r r_{\text {mod }}+\varepsilon^{2}\right) .
\end{aligned}
$$

The modeling error $\mathrm{err}_{\text {mod }}$ can be further analyzed under appropriate assumptions about the structure of $a^{\varepsilon}$, such as local periodicity or random stationarity (see (4.12)).

4.3. Proof of the main results. We shall now proceed with the proofs of Theorems 4.4 and 4.5 from the previous section. To do so, we first let $\pi_{H} u^{0}$ denote the elliptic projection

$B_{H}\left(\pi_{H} u^{0}, v_{H}\right)=B^{0}\left(u^{0}, v_{H}\right)+\left(\partial_{t t} u^{0}, v_{H}\right)-\left(I_{H}\left(\partial_{t t} u^{0}\right), v_{H}\right)_{Q} \quad \forall v_{H} \in S^{\ell}\left(\Omega, \mathcal{T}_{H}\right)$,

where $B_{H}(\cdot, \cdot)$ is defined by $(3.12)$. For higher derivatives, the projection $\pi_{H}\left(\partial_{t}^{k} u^{0}\right)$ is defined accordingly. Since $B_{H}$ is coercive and bounded, and the right-hand side of (4.17) is linear in $v_{H}$, the projection $\pi_{H} u^{0} \in S^{\ell}\left(\Omega, \mathcal{T}_{H}\right)$ is uniquely defined due to the Lax-Milgram theorem. Moreover, since $B_{H}$ and $B^{0}$ do not depend on time, we have

$$
\partial_{t}^{k}\left(\pi_{H} u^{0}\right)=\pi_{H}\left(\partial_{t}^{k} u^{0}\right),
$$

Copyright (c) by SIAM. Unauthorized reproduction of this article is prohibited. 
provided that there is sufficient regularity. Note that if we set $v_{H}=\pi_{H} u^{0}$ in (4.17) and use the coercivity and boundedness of $B_{H}$, Lemma 4.2, (4.6), and standard interpolation results and assume sufficient regularity of $u^{0}$ (e.g., $u^{0} \in L^{2}\left(0, T ; H^{1}(\Omega)\right), \partial_{t}^{k} u^{0} \in$ $\left.L^{2}\left(0, T ; L^{2}(\Omega)\right), k=1,2\right)$, we obtain

$$
\left\|\pi_{H} u^{0}\right\|_{L^{2}\left(0, T ; H^{1}(\Omega)\right)} \leq C .
$$

In Lemmas 4.6 and 4.7 below, we establish bounds on the difference between $u^{0}$ and its projection $\pi_{H} u^{0}$. They are later used in the proofs of Theorems 4.4 and 4.5.

Lemma 4.6. Suppose that (4.2)-(4.6) hold for $\mu=0$ and that (4.7) holds. Moreover, assume that

$$
\begin{aligned}
& \partial_{t}^{k} u^{0} \in L^{2}\left(0, T ; H^{\ell+1}(\Omega)\right), \quad \partial_{t}^{2+k} u^{0} \in L^{2}\left(0, T ; H^{\ell}(\Omega)\right), \quad k=0,1,2, \\
& a_{i, j}^{0} \in W^{\ell+1, \infty}(\Omega), \quad i, j=1, \ldots, d .
\end{aligned}
$$

Then

$$
\left\|\partial_{t}^{k} u^{0}-\pi_{H}\left(\partial_{t}^{k} u^{0}\right)\right\|_{L^{2}\left(0, T ; H^{1}(\Omega)\right)} \leq C\left(H^{\ell}+e_{H M M}+\varepsilon^{2}\right) .
$$

Proof. We now prove (4.19) for $k=0$. For higher $k$, the proof follows by differentiating (4.17).

Starting from (4.17), we first derive the estimate

$$
\begin{aligned}
B_{H}\left(\pi_{H} u^{0}-I_{H} u^{0}, v_{H}\right)= & B^{0}\left(u^{0}-I_{H} u^{0}, v_{H}\right)+B^{0}\left(I_{H} u^{0}, v_{H}\right)-B_{H}^{0}\left(I_{H} u^{0}, v_{H}\right) \\
& +B_{H}^{0}\left(I_{H} u^{0}, v_{H}\right)-B_{H}\left(I_{H} u^{0}, v_{H}\right) \\
& +\left(\partial_{t t} u^{0}-I_{H}\left(\partial_{t t} u^{0}\right), v_{H}\right)-\left(I_{H}\left(\partial_{t t} u^{0}\right), v_{H}\right)_{M} \\
& +\left(I_{H}\left(\partial_{t t} u^{0}\right), v_{H}\right)-\left(I_{H}\left(\partial_{t t} u^{0}\right), v_{H}\right)_{H} \\
\leq & C H^{\ell}\left(\Lambda+\max _{i, j}\left\|a_{i j}^{0}\right\|_{W^{\ell, \infty}}\right)\left\|u^{0}\right\|_{H^{\ell+1}}\left\|v_{H}\right\|_{H^{1}} \\
& +C e_{\mathrm{HMM}}\left\|u^{0}\right\|_{H^{1}}\left\|v_{H}\right\|_{H^{1}}+C H^{\ell}\left\|\partial_{t t} u^{0}\right\|_{H^{\ell}}\left\|v_{H}\right\|_{H^{1}} \\
& +C \varepsilon^{2}\left\|\partial_{t t} u^{0}\right\|_{H^{1}}\left\|v_{H}\right\|_{H^{1}},
\end{aligned}
$$

where we have used the boundedness of $B^{0}$, Lemma 4.1, (4.2), (4.7), and Lemma 4.2. Next we set $v_{H}=\pi_{H} u^{0}-I_{H} u^{0}$ and use the coercivity of $B_{H}$. By integrating the resulting expression from 0 to $T$, we obtain

$$
\begin{aligned}
\left\|\pi_{H} u^{0}-I_{H} u^{0}\right\|_{L^{2}\left(H^{1}\right)} \leq C & \left(H^{\ell}\left(\left\|u^{0}\right\|_{L^{2}\left(H^{\ell+1}\right)}+\left\|\partial_{t t} u^{0}\right\|_{L^{2}\left(H^{\ell}\right)}\right)+e_{\mathrm{HMM}}\left\|u^{0}\right\|_{L^{2}\left(H^{1}\right)}\right. \\
& \left.+\varepsilon^{2}\left\|\partial_{t t} u^{0}\right\|_{L^{2}\left(H^{1}\right)}\right) .
\end{aligned}
$$

Finally, the triangle inequality yields

$$
\left\|\pi_{H} u^{0}-u^{0}\right\|_{L^{2}\left(H^{1}\right)} \leq\left\|\pi_{H} u^{0}-I_{H} u^{0}\right\|_{L^{2}\left(H^{1}\right)}+\left\|I_{H} u^{0}-u^{0}\right\|_{L^{2}\left(H^{1}\right)} .
$$

Together with (4.7) to estimate the last term in (4.20), this concludes the proof.

Lemma 4.7. Suppose that (4.2)-(4.6) hold for $\mu=1$ and that (4.7) holds. Assume in addition that

$$
\begin{aligned}
\partial_{t}^{k} u^{0} & \in L^{2}\left(0, T ; H^{\ell+1}(\Omega)\right), \quad \partial_{t}^{2+k} u^{0} \in L^{2}\left(0, T ; H^{\ell+1}(\Omega)\right), & & k=0,1, \\
a_{i, j}^{0} & \in W^{\ell+1, \infty}(\Omega), & & i, j=1, \ldots, d .
\end{aligned}
$$

Copyright $@$ by SIAM. Unauthorized reproduction of this article is prohibited. 
Then

$$
\left\|\partial_{t}^{k} u^{0}-\pi_{H}\left(\partial_{t}^{k} u^{0}\right)\right\|_{L^{2}\left(0, T ; L^{2}(\Omega)\right)} \leq C\left(H^{\ell+1}+e_{H M M}+\varepsilon^{2}\right)
$$

for $H \leq H_{0}$.

Proof. Again we show only the proof for $k=0$. For higher $k$, the proof follows by differentiation.

Following a standard Aubin-Nitsche duality argument, we let $\varphi_{g}(t) \in H_{0}^{1}(\Omega)$ be the solution of

$$
B^{0}\left(v, \varphi_{g}(t)\right)=(v, g(t)) \quad \forall v \in H_{0}^{1}(\Omega),
$$

for any $g \in L^{2}\left(0, T ; L^{2}(\Omega)\right)$. Our regularity assumptions then imply that

$$
\varphi_{g} \in L^{2}\left(0, T ; H^{2}(\Omega) \cap H_{0}^{1}(\Omega)\right) \quad \text { and } \quad\left\|\varphi_{g}\right\|_{L^{2}\left(H^{2}\right)} \leq C\|g\|_{L^{2}\left(L^{2}\right)} .
$$

We now set $v=\pi_{H} u^{0}-u^{0}$ in (4.22) and use (4.17) to obtain

$$
\begin{aligned}
\left(\pi_{H} u^{0}-u^{0}, g\right)= & B^{0}\left(\pi_{H} u^{0}-u^{0}, \varphi_{g}\right)-B_{H}\left(\pi_{H} u^{0}, v_{H}\right)+B^{0}\left(u^{0}, v_{H}\right) \\
& +\left(\partial_{t t} u^{0}, v_{H}\right)-\left(I_{H}\left(\partial_{t t} u^{0}\right), v_{H}\right)_{Q} \\
= & B^{0}\left(\pi_{H} u^{0}-u^{0}, \varphi_{g}-v_{H}\right) \\
& +B^{0}\left(\pi_{H} u^{0}-I_{H} u^{0}, v_{H}\right)-B_{H}\left(\pi_{H} u^{0}-I_{H} u^{0}, v_{H}\right) \\
& +B^{0}\left(I_{H} u^{0}, v_{H}\right)-B_{H}\left(I_{H} u^{0}, v_{H}\right) \\
& +\left(\partial_{t t} u^{0}-I_{H}\left(\partial_{t t} u^{0}\right), v_{H}\right)+\left(I_{H}\left(\partial_{t t} u^{0}\right), v_{H}\right)-\left(I_{H}\left(\partial_{t t} u^{0}\right), v_{H}\right)_{H} \\
& -\left(I_{H}\left(\partial_{t t} u^{0}\right), v_{H}\right)_{M} .
\end{aligned}
$$

Next, we set $v_{H}=I_{H} \varphi_{g}$ and integrate from 0 to $T$, which yields

$$
\begin{aligned}
& \left|\int_{0}^{T}\left(\pi_{H} u^{0}-u^{0}, g,\right) d t\right| \leq \int_{0}^{T}\left|B^{0}\left(\pi_{H} u^{0}-u^{0}, \varphi_{g}-I_{H} \varphi_{g}\right)\right| d t \\
& \quad+\int_{0}^{T}\left|B^{0}\left(\pi_{H} u^{0}-I_{H} u^{0}, I_{H} \varphi_{g}\right)-B_{H}\left(\pi_{H} u^{0}-I_{H} u^{0}, I_{H} \varphi_{g}\right)\right| d t \\
& \quad+\int_{0}^{T}\left|B^{0}\left(I_{H} u^{0}, I_{H} \varphi_{g}\right)-B_{H}\left(I_{H} u^{0}, I_{H} \varphi_{g}\right)\right| d t \\
& \quad+C H^{\ell+1}\left\|\partial_{t t} u^{0}\right\|_{L^{2}\left(H^{\ell+1}\right)}\left(\left\|\varphi_{g}\right\|_{L^{2}\left(L^{2}\right)}+\left\|\varphi_{g}\right\|_{L^{2}\left(H^{2}\right)}\right) \\
& \quad+C \varepsilon^{2}\left\|\partial_{t t} u^{0}\right\|_{L^{2}\left(H^{1}\right)}\left\|\varphi_{g}\right\|_{L^{2}\left(H^{1}\right)},
\end{aligned}
$$

where we have used (4.5) with $\mu=1,(4.7)$, and Lemma 4.2 to bound the last four terms.

We shall now estimate the three remaining integrals on the right-hand side of the above inequality. Since $B^{0}$ is bounded, we immediately deduce for the first integral that

$$
\begin{aligned}
\int_{0}^{T}\left|B^{0}\left(\pi_{H} u^{0}-u^{0}, \varphi_{g}-I_{H} \varphi_{g}\right)\right| d t & \leq C\left\|\pi_{H} u^{0}-u^{0}\right\|_{L^{2}\left(H^{1}\right)}\left\|\varphi_{g}-I_{H} \varphi_{g}\right\|_{L^{2}\left(H^{1}\right)} \\
& \leq C\left(H^{\ell+1}+e_{\mathrm{HMM}}+\varepsilon^{2}\right)\left\|\varphi_{g}\right\|_{L^{2}\left(H^{2}\right)}
\end{aligned}
$$

Copyright $@$ by SIAM. Unauthorized reproduction of this article is prohibited. 
for $H \leq H_{0}$. Here we have used Lemma 4.6, (4.7), and the fact that $\varphi_{g}(t) \in H^{2}(\Omega)$. For the second integral we have for $H \leq H_{0}$

$$
\begin{aligned}
\int_{0}^{T} \mid & B^{0}\left(\pi_{H} u^{0}-I_{H} u^{0}, I_{H} \varphi_{g}\right)-B_{H}\left(\pi_{H} u^{0}-I_{H} u^{0}, I_{H} \varphi_{g}\right) \mid d t \\
\leq & \int_{0}^{T}\left|B^{0}\left(\pi_{H} u^{0}-I_{H} u^{0}, I_{H} \varphi_{g}\right)-B_{H}^{0}\left(\pi_{H} u^{0}-I_{H} u^{0}, I_{H} \varphi_{g}\right)\right| d t \\
& \quad+\int_{0}^{T}\left|B_{H}^{0}\left(\pi_{H} u^{0}-I_{H} u^{0}, I_{H} \varphi_{g}\right)-B_{H}\left(\pi_{H} u^{0}-I_{H} u^{0}, I_{H} \varphi_{g}\right)\right| d t \\
\leq & C H\left\|\pi_{H} u^{0}-I_{H} u^{0}\right\|_{L^{2}\left(H^{1}\right)}\left\|\varphi_{g}\right\|_{L^{2}\left(H^{1}\right)} \\
& +C e_{\text {HMM }}\left\|\pi_{H} u^{0}-I_{H} u^{0}\right\|_{L^{2}\left(H^{1}\right)}\left\|\varphi_{g}\right\|_{L^{2}\left(H^{1}\right)} \\
\leq & C\left(H^{\ell+1}+e_{\mathrm{HMM}}+\varepsilon^{2}\right)\left\|\varphi_{g}\right\|_{L^{2}\left(H^{2}\right)} .
\end{aligned}
$$

Here we first used (4.3) and Lemma 4.1, and then Lemma 4.6 together with (4.18) for $\pi_{H} u^{0}$ and a similar bound for $I_{H} u^{0}$.

To derive an upper bound for the third integral, we again use Lemma 4.1 and (4.2) with $\mu=1$, which yields

$$
\begin{aligned}
\int_{0}^{T} \mid & B^{0}\left(I_{H} u^{0}, I_{H} \varphi_{g}\right)-B_{H}\left(I_{H} u^{0}, I_{H} \varphi_{g}\right) \mid d t \\
\leq & \int_{0}^{T}\left|B^{0}\left(I_{H} u^{0}, I_{H} \varphi_{g}\right)-B_{H}^{0}\left(I_{H} u^{0}, I_{H} \varphi_{g}\right)\right| d t \\
& \quad+\int_{0}^{T}\left|B_{H}^{0}\left(I_{H} u^{0}, I_{H} \varphi_{g}\right)-B_{H}\left(I_{H} u^{0}, I_{H} \varphi_{g}\right)\right| d t \\
\leq & C\left(H^{\ell+1}+e_{\mathrm{HMM}}\right)\left\|\varphi_{g}\right\|_{L^{2}\left(H^{2}\right)} .
\end{aligned}
$$

Finally, we combine the upper bounds derived above with (4.23) to estimate the numerator in

$$
\left\|\pi_{H} u^{0}-u^{0}\right\|_{L^{2}\left(L^{2}\right)}=\sup _{g \in L^{2}\left(L^{2}\right) g \neq 0} \frac{\left|\int_{0}^{T}\left(\pi_{H} u^{0}-u^{0}, g\right) d t\right|}{\|g\|_{L^{2}\left(L^{2}\right)}},
$$

which yields (4.21) with $k=0$.

We are now in a position to prove our two main results stated in Theorems 4.4 and 4.5 .

Proof of Theorem 4.4. We consider $u^{0}$ and $u_{H}$, solutions of (2.5) and (3.11), respectively, and let $\zeta_{H}=u_{H}-\pi_{H} u^{0}$. Then, a direct calculation yields

$$
\begin{aligned}
& \left(\partial_{t t} \zeta_{H}, v_{H}\right)_{Q}+B_{H}\left(\zeta_{H}, v_{H}\right)=\left(F, v_{H}\right)-\left(\partial_{t t}\left(\pi_{H} u^{0}\right), v_{H}\right)_{Q}-B_{H}\left(\pi_{H} u^{0}, v_{H}\right) \\
& \quad=B^{0}\left(u^{0}, v_{H}\right)+\left(\partial_{t t} u^{0}, v_{H}\right)-\left(\pi_{H}\left(\partial_{t t} u^{0}\right), v_{H}\right)_{Q}-B_{H}\left(\pi_{H} u^{0}, v_{H}\right) \\
& \quad=\left(I_{H}\left(\partial_{t t} u^{0}\right)-\pi_{H}\left(\partial_{t t} u^{0}\right), v_{H}\right)_{Q}
\end{aligned}
$$

where we have used (4.17) for the last equality. Next, we set $v_{H}=\partial_{t} \zeta_{H}$ and exploit the symmetry of $(\cdot, \cdot)_{Q}$ and $B_{H}$ to rewrite $(4.24)$ as

$$
\frac{1}{2} \frac{d}{d t}\left(\left(\partial_{t} \zeta_{H}, \partial_{t} \zeta_{H}\right)_{Q}+B_{H}\left(\zeta_{H}, \zeta_{H}\right)\right)=\left(I_{H}\left(\partial_{t t} u^{0}\right)-\pi_{H}\left(\partial_{t t} u^{0}\right), \partial_{t} \zeta_{H}\right)_{Q} .
$$

Copyright $@$ ( ) by SIAM. Unauthorized reproduction of this article is prohibited. 
For $0<t<T$, we now let

$$
\eta(t)=\left(\partial_{t} \zeta_{H}, \partial_{t} \zeta_{H}\right)_{Q}+B_{H}\left(\zeta_{H}, \zeta_{H}\right)
$$

and use (3.13), Lemma 4.2, and Young's inequality to obtain

$$
\begin{aligned}
\frac{1}{2} \frac{d}{d t} \eta(t)= & \left(I_{H}\left(\partial_{t t} u^{0}\right)-\pi_{H}\left(\partial_{t t} u^{0}\right), \partial_{t} \zeta_{H}\right)_{H}+\left(I_{H}\left(\partial_{t t} u^{0}\right)-\pi_{H}\left(\partial_{t t} u^{0}\right), \partial_{t} \zeta_{H}\right)_{M} \\
\leq C & \left\|I_{H}\left(\partial_{t t} u^{0}\right)-\pi_{H}\left(\partial_{t t} u^{0}\right)\right\|_{L^{2}}\left\|\partial_{t} \zeta_{H}\right\|_{L^{2}} \\
& \left.\quad+\varepsilon^{2}\left\|\nabla\left(I_{H}\left(\partial_{t t} u^{0}\right)-\pi_{H}\left(\partial_{t t} u^{0}\right)\right)\right\|_{L^{2}}\left\|\nabla\left(\partial_{t} \zeta_{H}\right)\right\|_{L^{2}}\right) \\
\leq C & \left\|I_{H}\left(\partial_{t t} u^{0}\right)-\pi_{H}\left(\partial_{t t} u^{0}\right)\right\|_{L^{2}}^{2}+\left\|\partial_{t} \zeta_{H}\right\|_{L^{2}}^{2} \\
& +\left\|\nabla\left(I_{H}\left(\partial_{t t} u^{0}\right)-\pi_{H}\left(\partial_{t t} u^{0}\right)\right)\right\|_{L^{2}}^{2}+\varepsilon^{4} \| \nabla\left(\partial_{t} \zeta_{H} \|_{L^{2}}^{2}\right) .
\end{aligned}
$$

Since by assumption $\left\|u_{H}\right\|_{H^{1}\left(0, T ; H^{1}(\Omega)\right)} \leq c$ independently of $H$ and (4.18) holds, $\left\|\nabla\left(\partial_{t} \zeta_{H}\right)\right\|_{L^{2}}$ is also bounded independently of $H$ on $[0, T]$. Moreover, from (4.6) and Lemma 4.2, we deduce that

$$
\left\|\partial_{t} \zeta_{H}\right\|_{L^{2}}^{2} \leq C\left\|\partial_{t} \zeta_{H}\right\|_{H}^{2} \leq C\left(\left\|\partial_{t} \zeta_{H}\right\|_{H}^{2}+\left(\partial_{t} \zeta_{H}, \partial_{t} \zeta_{H}\right)_{M}\right)=C\left(\partial_{t} \zeta_{H}, \partial_{t} \zeta_{H}\right)_{Q} .
$$

Thus by adding $B_{H}\left(\zeta_{H}, \zeta_{H}\right) \geq 0$ to the right-hand side of inequality (4.25), we find

$$
\frac{1}{2} \frac{d}{d t} \eta(t) \leq C\left(\eta(t)+\left\|I_{H}\left(\partial_{t t} u^{0}\right)-\pi_{H}\left(\partial_{t t} u^{0}\right)\right\|_{H^{1}}^{2}+\varepsilon^{4}\right) .
$$

Gronwall's inequality then yields

$$
\sup _{0 \leq t \leq T} \eta(t) \leq C\left(\eta(0)+\left\|I_{H}\left(\partial_{t t} u^{0}\right)-\pi_{H}\left(\partial_{t t} u^{0}\right)\right\|_{L^{2}\left(H^{1}\right)}^{2}+\varepsilon^{4}\right) .
$$

For the second term on the right-hand side of (4.26), an upper bound immediately follows from Lemma 4.6 with $k=2$ :

$$
\left\|I_{H}\left(\partial_{t t} u^{0}\right)-\pi_{H}\left(\partial_{t t} u^{0}\right)\right\|_{L^{2}\left(H^{1}\right)}^{2} \leq C\left(H^{2 \ell}+e_{\mathrm{HMM}}^{2}+\varepsilon^{4}\right) .
$$

It remains to bound the first term on the right-hand side of (4.26). By Lemma 4.2 , we have

$$
\begin{aligned}
\eta(0) & =\left(\partial_{t} \zeta_{H}(0), \partial_{t} \zeta_{H}(0)\right)_{Q}+B_{H}\left(\zeta_{H}(0), \zeta_{H}(0)\right) \\
& \leq C\left(\left\|\partial_{t} \zeta_{H}(0)\right\|_{H}^{2}+\varepsilon^{2}\left\|\nabla\left(\partial_{t} \zeta_{H}(0)\right)\right\|_{L^{2}}^{2}+B_{H}\left(\zeta_{H}(0), \zeta_{H}(0)\right)\right) .
\end{aligned}
$$

We shall now estimate each term on the right-hand side of (4.27). For the last term, we easily derive the upper bound

$$
\begin{aligned}
\left|B_{H}\left(\zeta_{H}(0), \zeta_{H}(0)\right)\right| \leq & C\left\|\zeta_{H}(0)\right\|_{H^{1}}^{2}=C\left\|I_{H} f-\pi_{H} u^{0}(0)\right\|_{H^{1}}^{2} \\
\leq & C\left(\left\|I_{H} f-f\right\|_{H^{1}}^{2}+\left\|u^{0}(0)-\pi_{H} u^{0}(0)\right\|_{H^{1}}^{2}\right) \\
\leq & C\left(H^{2 \ell}\|f\|_{H^{\ell+1}}^{2}\right. \\
& \left.\quad \quad+\left\|u^{0}-\pi_{H} u^{0}\right\|_{L^{2}\left(H^{1}\right)}^{2}+\left\|\partial_{t} u^{0}-\pi_{H}\left(\partial_{t} u^{0}\right)\right\|_{L^{2}\left(H^{1}\right)}^{2}\right) \\
& \leq C\left(H^{2 \ell}+e_{\mathrm{HMM}}^{2}+\varepsilon^{4}\right),
\end{aligned}
$$

Copyright (c) by SIAM. Unauthorized reproduction of this article is prohibited. 
where we have used (4.7), the embedding of $H^{1}\left(0, T ; H^{1}(\Omega)\right)$ into $C\left(0, T ; H^{1}(\Omega)\right)$, and Lemma 4.6. To estimate the first term on the right-hand side of (4.27), we use (4.6) and the continuous embedding of $H^{1}\left(0, T ; L^{2}(\Omega)\right)$ into $C\left(0, T ; L^{2}(\Omega)\right)$ :

$$
\begin{aligned}
\left\|\partial_{t} \zeta_{H}(0)\right\|_{H} \leq & c_{2}\left\|\partial_{t} \zeta_{H}(0)\right\|_{L^{2}}=c_{2}\left\|I_{H} g-\pi_{H}\left(\partial_{t} u^{0}(0)\right)\right\|_{L^{2}} \\
\leq & c_{2}\left(\left\|I_{H} g-g\right\|_{L^{2}}+\left\|\partial_{t} u^{0}(0)-\pi_{H}\left(\partial_{t} u^{0}(0)\right)\right\|_{L^{2}}\right) \\
\leq & C\left(H^{\ell}\|g\|_{H^{\ell}}\right. \\
& \left.\quad+\left\|\partial_{t} u^{0}-\pi_{H}\left(\partial_{t} u^{0}\right)\right\|_{L^{2}\left(L^{2}\right)}+\left\|\partial_{t t} u^{0}-\pi_{H}\left(\partial_{t t} u^{0}\right)\right\|_{L^{2}\left(L^{2}\right)}\right) \\
\leq & C\left(H^{\ell}+e_{\mathrm{HMM}}+\varepsilon^{2}\right) .
\end{aligned}
$$

Similarly we infer that the second term is bounded as

$$
\left\|\nabla\left(\partial_{t} \zeta_{H}(0)\right)\right\|_{L^{2}} \leq C\left(H^{\max (1, \ell-1)}+e_{\mathrm{HMM}}+\varepsilon^{2}\right) .
$$

By combining the above three estimates with (4.27), we thus conclude that

$$
\eta(0) \leq C\left(H^{2 \ell}+e_{\mathrm{HMM}}^{2}+\varepsilon^{4}+\varepsilon^{2}\left(H^{\max (2,2 \ell-2)}+e_{\mathrm{HMM}}^{2}+\varepsilon^{4}\right)\right),
$$

which reduces to

$$
\eta(0) \leq C\left(H^{2 \ell}+e_{\mathrm{HMM}}^{2}+\varepsilon^{4}\right) .
$$

Finally, we split the quantity of interest as

$$
\left\|u^{0}-u_{H}\right\| \leq\left\|u^{0}-\pi_{H} u^{0}\right\|+\left\|\zeta_{H}\right\|
$$

and use Lemma 4.6 to bound the first term on the right. To bound the last term, the continuous embeddings of $H^{1}\left(0, T ; H^{1}(\Omega)\right)$ into $C\left(0, T ; H^{1}(\Omega)\right)$ and $H^{1}\left(0, T ; L^{2}(\Omega)\right)$ into $C\left(0, T ; L^{2}(\Omega)\right)$ imply

$$
c\left(\left\|\partial_{t} \zeta_{H}\right\|_{L^{\infty}\left(L^{2}\right)}^{2}+\left\|\zeta_{H}\right\|_{L^{\infty}\left(H^{1}\right)}^{2}\right) \leq \sup _{0 \leq t \leq T} \eta(t),
$$

which together with (4.26) concludes the proof.

Proof of Theorem 4.5. Following the proof of Theorem 4.4, we let $\zeta_{H}=u_{H}-\pi_{H} u^{0}$ and recall from (4.24) that

$$
\left(\partial_{t t} \zeta_{H}, v_{H}\right)_{Q}+B_{H}\left(\zeta_{H}, v_{H}\right)=\left(I_{H}\left(\partial_{t t} u^{0}\right)-\pi_{H}\left(\partial_{t t} u^{0}\right), v_{H}\right)_{Q} .
$$

Next, we define

$$
\Psi_{H}=I_{H}\left(\partial_{t} u^{0}\right)-\pi_{H}\left(\partial_{t} u^{0}\right)-\partial_{t} \zeta_{H}, \quad \Phi_{H}=I_{H}\left(\partial_{t} u^{0}\right)-\pi_{H}\left(\partial_{t} u^{0}\right)
$$

and rewrite (4.28) as

$$
\begin{aligned}
-\left(\partial_{t} \zeta_{H}, \partial_{t} v_{H}\right)_{Q}+B_{H}\left(\zeta_{H}, v_{H}\right) & =-\left(\partial_{t} \zeta_{H}, \partial_{t} v_{H}\right)_{Q}+\left(\partial_{t} \Psi_{H}, v_{H}\right)_{Q} \\
& =-\left(\partial_{t} \zeta_{H}, \partial_{t} v_{H}\right)_{Q}+\frac{d}{d t}\left(\Psi_{H}, v_{H}\right)_{Q}-\left(\Psi_{H}, \partial_{t} v_{H}\right)_{Q} \\
& =\frac{d}{d t}\left(\Psi_{H}, v_{H}\right)_{Q}-\left(\Phi_{H}, \partial_{t} v_{H}\right)_{Q}
\end{aligned}
$$

Copyright $@$ by SIAM. Unauthorized reproduction of this article is prohibited. 
for all $v_{H} \in L^{2}\left(0, T ; S^{\ell}\left(\Omega, \mathcal{T}_{H}\right)\right)$ with $\partial_{t} v_{H} \in L^{2}\left(0, T ; S^{\ell}\left(\Omega, \mathcal{T}_{H}\right)\right)$.

For fixed $s \leq T$, we now set

$$
v_{H}(t)=\int_{t}^{s} \zeta_{H}(\tau) d \tau
$$

and use $\partial_{t} v_{H}=-\zeta_{H}$ to infer that

$$
\frac{1}{2} \frac{d}{d t}\left(\left(\zeta_{H}, \zeta_{H}\right)_{Q}-B_{H}\left(v_{H}, v_{H}\right)\right)=\frac{d}{d t}\left(\Psi_{H}, v_{H}\right)_{Q}+\left(\Phi_{H}, \zeta_{H}\right)_{Q} .
$$

Integration from 0 to $s$ then yields

$$
\frac{1}{2}\left(\left(\zeta_{H}(s), \zeta_{H}(s)\right)_{Q}-\left(\zeta_{H}(0), \zeta_{H}(0)\right)_{Q}+B_{H}\left(v_{H}(0), v_{H}(0)\right)\right)=\int_{0}^{s}\left(\Phi, \zeta_{H}\right)_{Q} d t,
$$

because $v_{H}(s)=\Psi_{H}(0)=0$. Moreover, Lemma 4.1 implies that $B_{H}\left(v_{H}(0), v_{H}(0)\right)$ is positive. By using Lemma 4.2, the Cauchy-Schwarz inequality, Young's inequality, and (4.6), we thus obtain

$$
\begin{aligned}
\left\|\zeta_{H}(s)\right\|_{L^{2}}^{2} \leq & C\left(\left\|\zeta_{H}(0)\right\|_{L^{2}}^{2}+\varepsilon^{2}\left\|\nabla \zeta_{H}(0)\right\|_{L^{2}}^{2}\right) \\
& +\frac{C}{\kappa}\left\|I_{H}\left(\partial_{t} u^{0}\right)-\pi_{H}\left(\partial_{t} u^{0}\right)\right\|_{L^{2}\left(L^{2}\right)}^{2}+C \kappa\left\|\zeta_{H}\right\|_{L^{\infty}\left(L^{2}\right)}^{2} \\
& +C \varepsilon^{2}\left(\left\|I_{H}\left(\partial_{t} u^{0}\right)-\pi_{H}\left(\partial_{t} u^{0}\right)\right\|_{L^{2}\left(H^{1}\right)}^{2}+\left\|\zeta_{H}\right\|_{L^{2}\left(H^{1}\right)}^{2}\right)
\end{aligned}
$$

for any $\kappa>0$. Taking the supremum over $s$ now yields, for $\kappa$ sufficiently small,

$$
\begin{aligned}
\left\|\zeta_{H}\right\|_{L^{\infty}\left(L^{2}\right)}^{2} \leq & C\left(\left\|\zeta_{H}(0)\right\|_{L^{2}}^{2}+\left\|I_{H}\left(\partial_{t} u^{0}\right)-\pi_{H}\left(\partial_{t} u^{0}\right)\right\|_{L^{2}\left(L^{2}\right)}^{2}\right) \\
& +C \varepsilon^{2}\left(\left\|\nabla \zeta_{H}(0)\right\|_{L^{2}}^{2}+\left\|I_{H}\left(\partial_{t} u^{0}\right)-\pi_{H}\left(\partial_{t} u^{0}\right)\right\|_{L^{2}\left(H^{1}\right)}^{2}+\left\|\zeta_{H}\right\|_{L^{2}\left(H^{1}\right)}^{2}\right) .
\end{aligned}
$$

To complete the proof, we must now estimate each term on the right-hand side of (4.29). For the first term, we easily find using (4.7) that

$$
\begin{aligned}
\left\|\zeta_{H}(0)\right\|_{L^{2}} & \leq\left\|\pi_{H} u^{0}(0)-u^{0}(0)\right\|_{L^{2}}+\left\|u^{0}(0)-I_{H} u^{0}(0)\right\|_{L^{2}} \\
& \leq C\left(H^{\ell+1}+e_{\mathrm{HMM}}+\varepsilon^{2}\right) .
\end{aligned}
$$

Similarly, the second and fourth terms on the right-hand side of (4.29) are immediately estimated by Lemmas 4.6 and 4.7. To derive an upper bound for the third term, we use the continuous embedding from $H^{1}\left(0, T ; H^{1}(\Omega)\right)$ into $C\left(0, T ; H^{1}(\Omega)\right)$ and then Lemma 4.6, as follows:

$$
\begin{aligned}
\left\|\nabla \zeta_{H}(0)\right\|_{L^{2}} & \leq\left\|I_{H} f-u^{0}(0)\right\|_{H^{1}}+\left\|u^{0}(0)-\pi_{H} u^{0}(0)\right\|_{H^{1}} \\
& \leq C\left(H^{\ell}+\left\|u^{0}-\pi_{H} u^{0}\right\|_{L^{2}\left(H^{1}\right)}+\left\|\partial_{t} u^{0}-\pi_{H}\left(\partial_{t} u^{0}\right)\right\|_{L^{2}\left(H^{1}\right)}\right) \\
& \leq C\left(H^{\ell}+e_{\mathrm{HMM}}+\varepsilon^{2}\right) .
\end{aligned}
$$

The remaining last term in (4.29) is bounded above independently of $H$ due to $\left\|u_{H}\right\|_{L^{2}\left(0, T ; H^{1}(\Omega)\right)} \leq c$ and (4.18). Finally, we combine the above estimates for (4.29) with an argument similar to that used at the end of the proof of Theorem 4.4, which completes the proof.

Copyright $@$ by SIAM. Unauthorized reproduction of this article is prohibited. 
5. Numerical experiments. We shall now demonstrate the accuracy and usefulness of our FE-HMM-L scheme during both finite- and long-time regimes. First, we validate the optimal convergence rates of Theorems 4.4 and 4.5 for a one-dimensional periodic model problem, where an exact solution is available for comparison. Our FE-HMM-L scheme, however, is not restricted to periodic media, as shown in a second example where the oscillatory tensor is not uniformly periodic. We also illustrate the versatility of the FE-HMM-L scheme by applying it to a two-dimensional problem with complex geometry. Then, we demonstrate the accuracy of FE-HMM-L for long-time simulations, when dispersive effects induced by the microstructures in the medium can no longer be neglected. Neither classical homogenization nor the former FE-HMM scheme from [8] can capture these dispersive effects; hence, they are both inadequate for numerical wave propagation over long times.

For the spatial discretization, we use standard FEMs for the macro- and the microsolver. The resulting second-order system of ordinary differential equations is integrated in time with the second-order leapfrog scheme. Since the CFL condition on $\Delta t$ is dictated by $H$ and not by the micro mesh size $h$, much larger time steps are admissible than in a fully resolved numerical solution. This leads to an additional significant reduction in the computational effort. Clearly, other time discretization schemes, such as Runge-Kutta or multistep methods, can be used.

\subsection{Short-time regime.}

One-dimensional periodic medium. We consider (2.1) with $F \equiv 0$ in the interval $\Omega=[-1,1]$ with homogeneous Dirichlet boundary conditions. The highly oscillatory (squared) velocity field is given by

$$
a^{\varepsilon}(x)=\sqrt{2}+\sin \left(2 \pi \frac{x}{\varepsilon}\right) .
$$

Because of the simple structure of $a^{\varepsilon}$, it is possible to compute the constant homogenized wave speed $\sqrt{a^{0}}=1$. Hence the solution of the homogenized wave equation (2.5) with initial data $f(x)=\sin (\pi x)$ and $g(x)=0$ is given by $u^{0}(x, t)=\sin (\pi x) \cos (\pi t)$. We emphasize that FE-HMM-L is not restricted to periodic media, whereas $a^{0}$ cannot be calculated in general.

First we let $\varepsilon=2^{-11}$ and use $\mathcal{P}^{1}$ finite elements on a uniform macroscopic mesh $\mathcal{T}_{H}$ for the sequence of meshes $H=2^{-k}, k=2,3, \ldots, 8$. For numerical quadrature we use the trapezoidal rule which results in two microproblems per macro finite element. The microproblems, defined on the sampling domains $K_{\delta}$ of diameter $\delta=\varepsilon$ with periodic coupling conditions, are also discretized with $\mathcal{P}^{1}$ elements on a uniform micromesh with $h=\delta \cdot 2^{-k}$. For each macromesh, we set the time step $\Delta t=H / 8$ proportional to $H$ according to the CFL stability condition. In Figure 1, we show the $L^{2}$ - and $H^{1}$-errors between $u_{H}$ and $u^{0}$ at the final time $T=2.75$. As predicted by Theorems 4.4 and 4.5, we observe second-order convergence in the $L^{2}$-norm and first-order convergence in the $H^{1}$-norm.

To achieve optimal convergence, it is crucial to refine simultaneously the macroand the micromesh. Otherwise, if we fix the resolution of the microproblem while refining only at the macroscale, then FE-HMM-L fails to achieve optimal secondorder convergence, as shown in Figure 2. Indeed, if $h$ remains constant, then $e_{\mathrm{HMM}}$, which scales as $(h / \varepsilon)^{2 q}[1,3]$, eventually dominates in the error estimates (4.15) and (4.16).

The error bounds in Theorems 4.4 and 4.5 differ from those previously derived for the former FE-HMM [8, Theorem 4.3] mainly through their explicit quadratic 

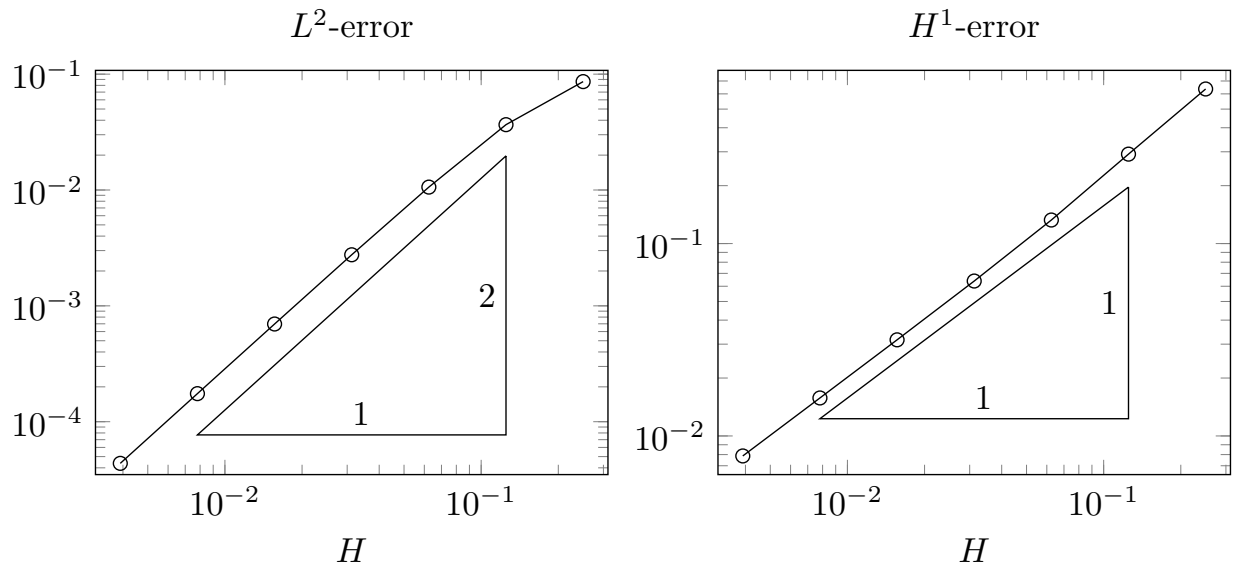

FIG. 1. One-dimensional periodic medium. $L^{2}$-error (left) and $H^{1}$-error (right) $\left\|u_{H}-u^{0}\right\|$ at time $T=2.75$, with simultaneous refinement of the macro mesh size $H$ and the micro mesh size $h$.
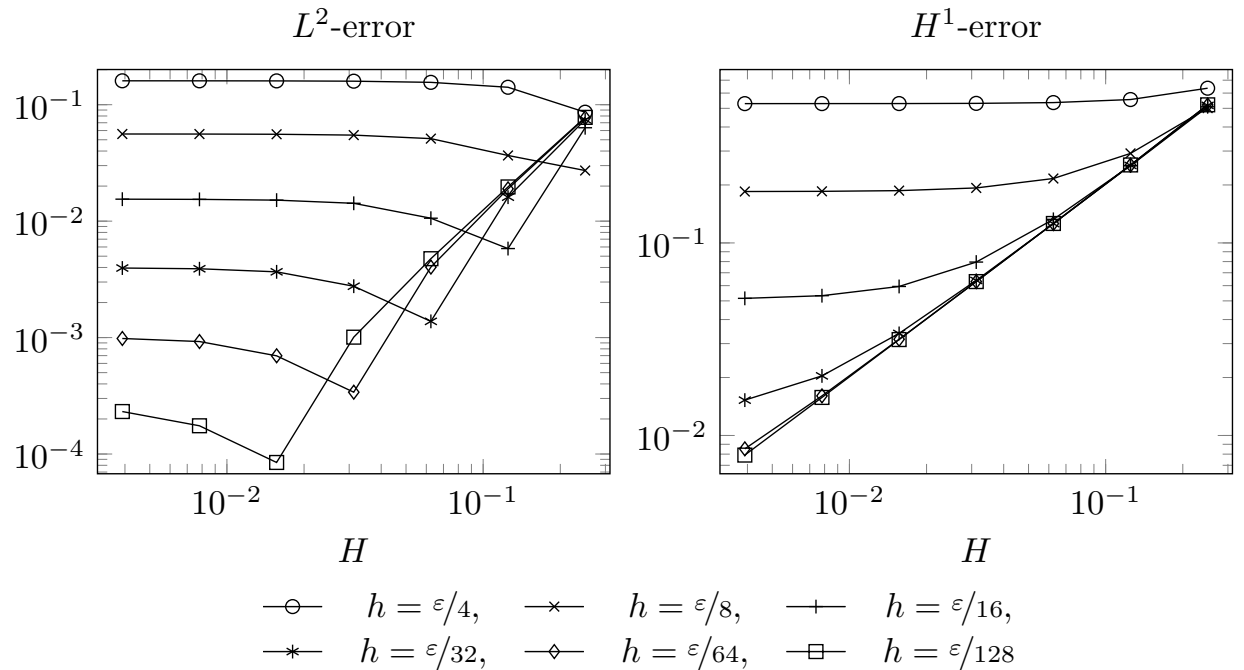

FIG. 2. One-dimensional periodic medium. $L^{2}$-error (left) and $H^{1}$-error (right) $\left\|u_{H}-u^{0}\right\|$ at time $T=2.75$, where only the macro mesh size $H$ is refined, but the micro mesh size $h$ is kept fixed. The different lines correspond to different values of $h$.

dependence on $\varepsilon$ in the case of FE-HMM-L. To exhibit this dependence, we choose a very fine macro- and micromesh with $H=2^{-11}$ and $h=\delta \cdot 2^{-10}$ to ensure that discretization errors are minimal. In the left frame of Figure 3, we observe the predicted second-order convergence with respect to $\varepsilon$. Note that only the $L^{2}$-error is shown, since the $H^{1}$-error behaves similarly.

For this simple periodic example, the choice $\delta=\varepsilon$ for the size of the sampling domain is quite obvious. In practice, however, the precise value of $\varepsilon$ may vary or be unknown. Still, the FE-HMM-L scheme can be applied. To illustrate this fact, we fix the values $\varepsilon=1 / 100, H=2^{-6}$, and $h=\delta \cdot 2^{-6}$, but let $\delta$ vary. Clearly $\delta \geq \varepsilon$ is needed to obtain reliable results, as the microproblems must cover at least one period in the microstructure. In the right frame of Figure 3, we observe that overestimating $\varepsilon$ does 

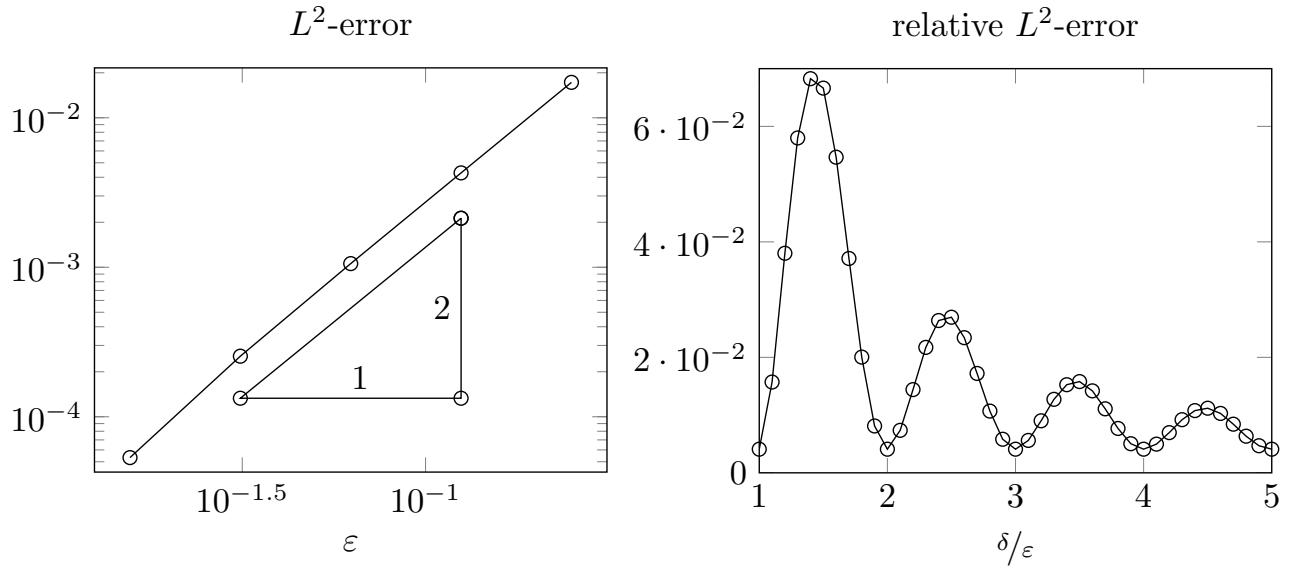

FIG. 3. One-dimensional periodic medium. Error $\left\|u_{H}-u^{0}\right\|$ at time $T=2.75$. Left: $L^{2}$-error versus the period $\varepsilon$. Right: Relative $L^{2}$-error versus the ratio between $\delta$ and $\varepsilon$.

not dramatically increase the relative $L^{2}$-error, while, as expected, the best results are achieved if $\delta$ is a multiple of $\varepsilon$.

One-dimensional nonperiodic medium. To show that FE-HMM-L also applies to more general media, we now consider (2.1) with $\Omega=[0,3], f(x)=g(x)=0$, and no source. At $x=1$, we impose a homogeneous Dirichlet condition, whereas at $x=0$ we impose the time-dependent boundary condition

$$
u^{\varepsilon}(0, t)= \begin{cases}\frac{1-\cos (4 \pi t)}{2} & \text { for } t \leq 0.5 \\ 0 & \text { for } t>0.5\end{cases}
$$

which corresponds to a left-incoming sinusoidal pulse. The (squared) velocity is given by

$$
a^{\varepsilon}(x)=1+0.2 \sin \left(2 \pi \frac{(x+0.3)^{2}}{\varepsilon}\right)(1+0.4 \sin (2 \pi x))+\tanh \left(\frac{0.5}{x+0.6}\right),
$$

with $\varepsilon=0.002$. As shown in Figure $4, a^{\varepsilon}(x)$ is highly oscillatory but nonperiodic: both the amplitude and the phase of the oscillations vary with distance, while the fast and slow scale dependencies do not explicitly separate.

In Figure 5, we compare the FE-HMM-L solution and an FEM solution, both computed on the same coarse mesh, with a reference solution computed on a finer mesh that fully resolves the oscillations of $a^{\varepsilon}$; see Figure 5. For the FE-HMM-L solution we use $H=1 / 150, \delta=1 / 300$, and piecewise linear FEs for both the macroand the microsolver. Although the convergence theory from the previous section does not apply to this very general medium, we observe that the FE-HMM-L approximates well the macroscopic behavior of the true solution.

Two-dimensional layered topography. Next, we consider (2.1) with $F \equiv 0$ in the two-dimensional domain $\Omega=[0,2] \times[-1,1]$ and set homogeneous Neumann boundary conditions on its entire boundary. The computational domain consists of four distinct subdomains, $\Omega_{i}, i=1, \ldots, 4$, shown in Figure 6. Inside each subdomain 

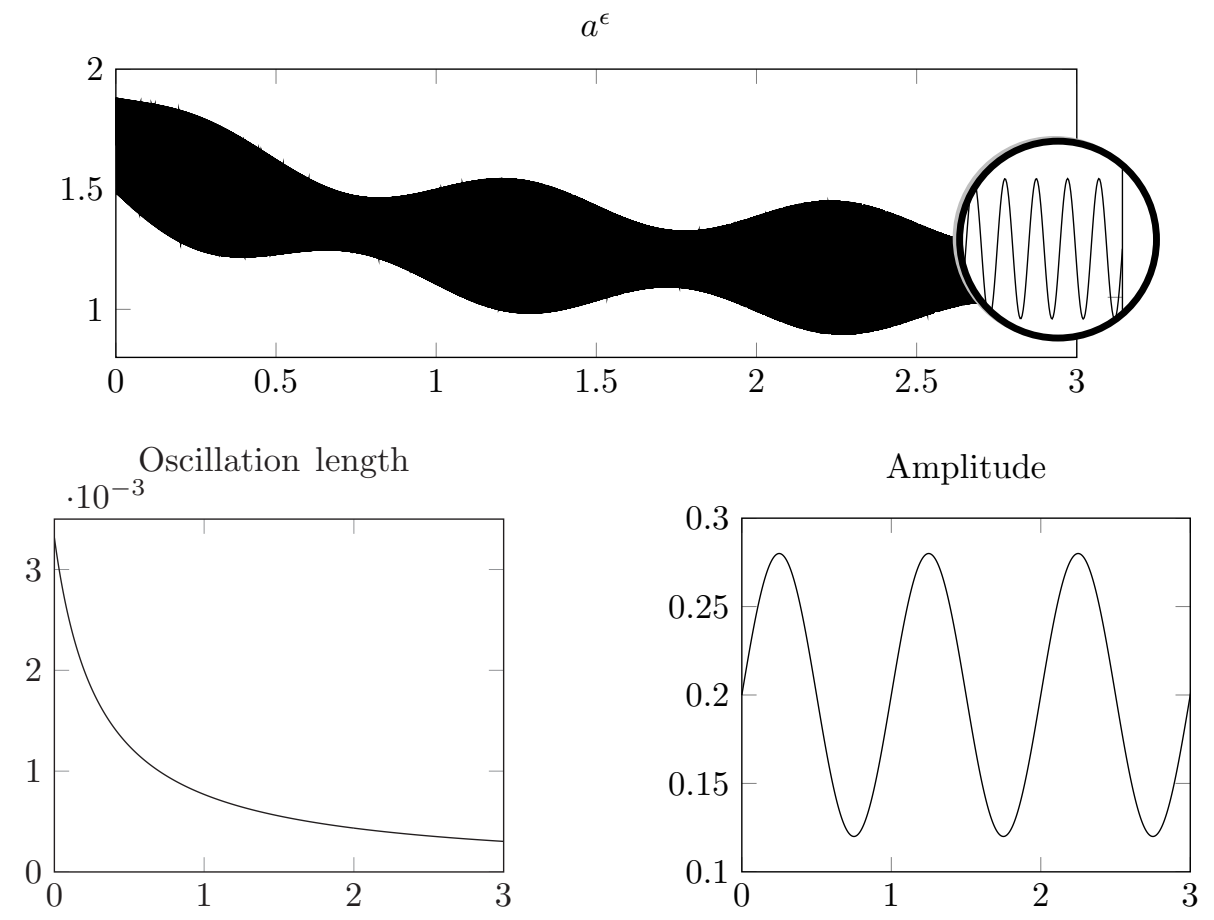

FIG. 4. One-dimensional nonperiodic medium. Top: Multiscale tensor $a^{\varepsilon}(x)$ for $\varepsilon=1 / 500$ with a zoom at $x=3$. Bottom: Change of the period (left) and the amplitude (right).

the (squared) velocity tensor $a^{\varepsilon}(x)$ varies in the vertical direction as

$$
a^{\varepsilon}(x)= \begin{cases}\left(\sqrt{5}+2 \sin \left(2 \pi \frac{x_{2}}{\varepsilon}\right)\right) I_{2 \times 2} & \text { for } x \in \Omega_{1}, \\ \left(\sqrt{5}+\sin \left(2 \pi \frac{x_{2}}{\varepsilon}\right)\right) I_{2 \times 2} & \text { for } x \in \Omega_{2}, \\ \left(\sqrt{2}+\frac{1}{2} \sin \left(2 \pi x_{2}\right)+\frac{1}{2} \sin \left(2 \pi \frac{x_{2}}{\varepsilon}\right)\right) I_{2 \times 2} & \text { for } x \in \Omega_{2}, \\ I_{2 \times 2} & \text { for } x \in \Omega_{4},\end{cases}
$$

where $\varepsilon=10^{-3}$ and $I_{2 \times 2}$ is the $2 \times 2$ identity matrix. The initial conditions $f, g$ are chosen to induce a downward moving plane wave with Gaussian profile, initially centered about $x_{2}=0.5$.

At the macroscale we use $\mathcal{P}^{1}$ FEs on a triangular mesh, which respects the discontinuities of $a^{\varepsilon}$ across interior interfaces, as shown in Figure 6. At the microscale we use $\mathcal{Q}^{1}$ FEs on square sampling domains of size $\delta=\varepsilon=10^{-3}$. Note that if the same micro mesh size $h$ was used everywhere throughout $\Omega$, the FE mesh would contain about 400 million instead of 65,526 elements at the macroscale.

In Figure 7, snapshots of the FE-HMM-L solution $u_{H}$ are shown at three different times. For comparison, we also display the numerical solution of the homogenized wave equation (2.5) with $a^{0}$ computed analytically, but also that with $a^{0}$ replaced by a simple locally averaged medium. Both $u_{H}$ and $u^{0}$ coincide as the initial Gaussian pulse propagates across the medium while generating multiple reflections at the interfaces. In contrast, the solution with a "naively" averaged medium displays errors in both phase and amplitude. In particular, it completely misses the interface between $\Omega_{1}$ and $\Omega_{2}$, where the amplitude but not the mean of the oscillations in $a^{\varepsilon}$ changes. 

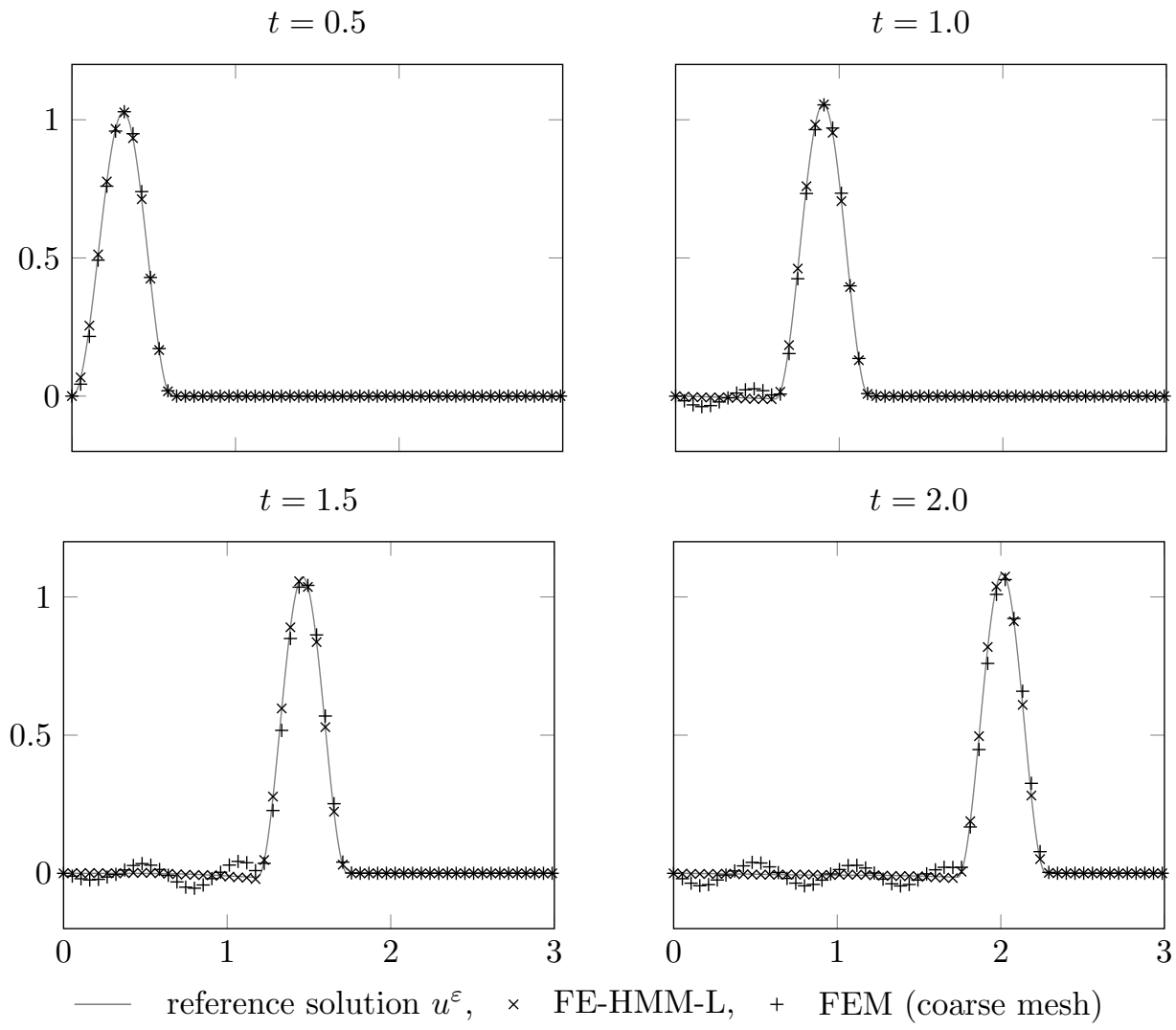

FIG. 5. One-dimensional nonperiodic medium. Snapshots of the reference, the FE-HMM-L, and the FEM solution on a coarse mesh at times $T=0.5,1.0,1.5,2.0$. The (unresolved) FEM solution deviates from the (true) fully resolved reference solution, whereas the FE-HMM-L, computed on the same coarse mesh, approximates well its macroscopic behavior.
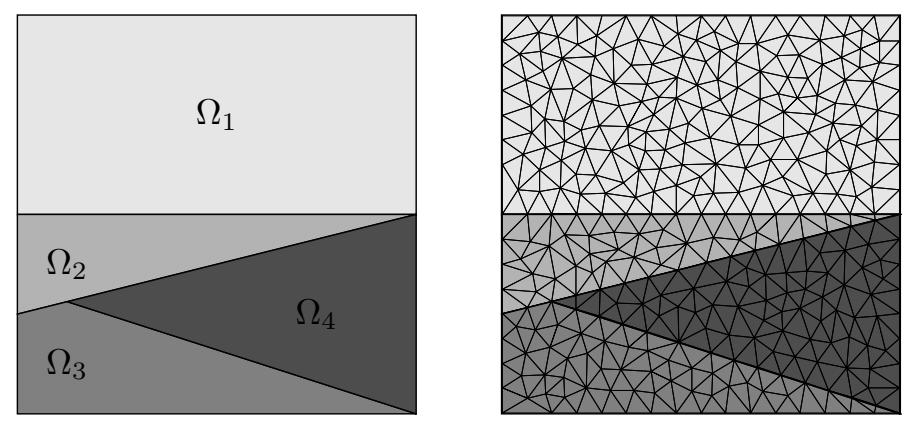

FIG. 6. Two-dimensional layered topography. Left: The computational domain $\Omega$ with its subdomains. Right: A sample triangulation of $\Omega$ which respects the inner interfaces.

5.2. Long-time regime. For short times, the solution $u^{0}$ of the homogenized wave equation (2.5) yields a good approximation of the true solution $u^{\varepsilon}$. At later times, however, dispersive wave trains develop, which are not captured by $u^{0}$. Not surprisingly, since the FE-HMM scheme presented in [8] is based on (2.5), its solution 
FE-HMM-L
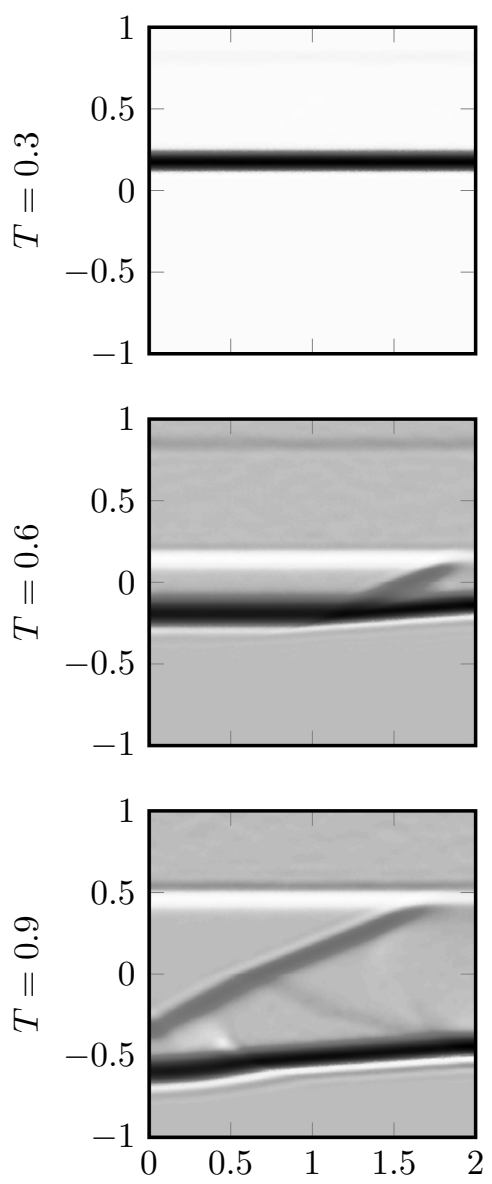

homogenized
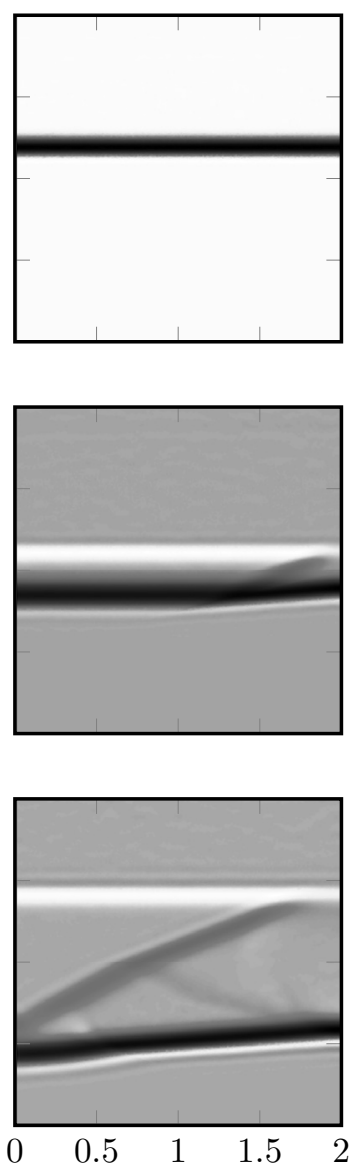

averaged
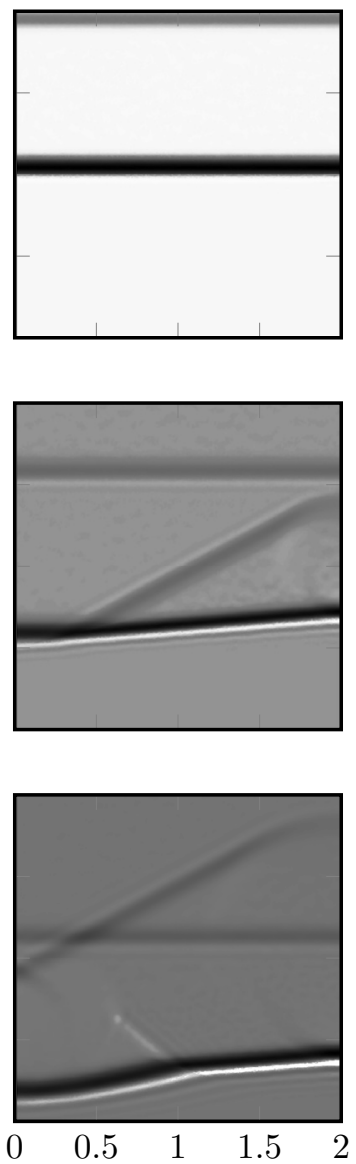

FIG. 7. Two-dimensional layered topography. Snapshots of the FE-HMM-L solution $u_{H}$ (left), the solution $u^{0}$ of the homogenized equation (2.5) (middle), and that with a locally averaged tensor (right) are shown at times $T=0.3,0.6,0.9$.

is also unable to reproduce those dispersive effects. In contrast, the FE-HMM-L method is based on the Boussinesq equation (1.3), which admits dispersive solutions, and thus it is indeed able to capture that dispersive behavior.

One-dimensional periodic medium. We consider again the one-dimensional periodic medium from section 5.1 with $\varepsilon=1 / 50$, but now impose periodic boundary conditions on $\Omega=[-1,1]$. As initial data, we choose the Gaussian pulse

$$
f(x)=\exp \left(\frac{-x^{2}}{\sigma^{2}}\right)
$$

with $\sigma^{2}=1 / 100$ and $g(x)=0$. The initial pulse splits into a left- and a right-moving wave, which meet again at $x=0$ every time $T=2,4,6, \ldots$, because of periodicity. Since the homogenized wave equation (2.5) has constant velocity $a^{0} \equiv 1$, its solution $u^{0}$ coincides with the initial condition at every even integer time $T=2,4,6, \ldots$. However, the true solution $u^{\varepsilon}$, computed with a fully resolved FEM, deviates from 
homogenized and FE-HMM

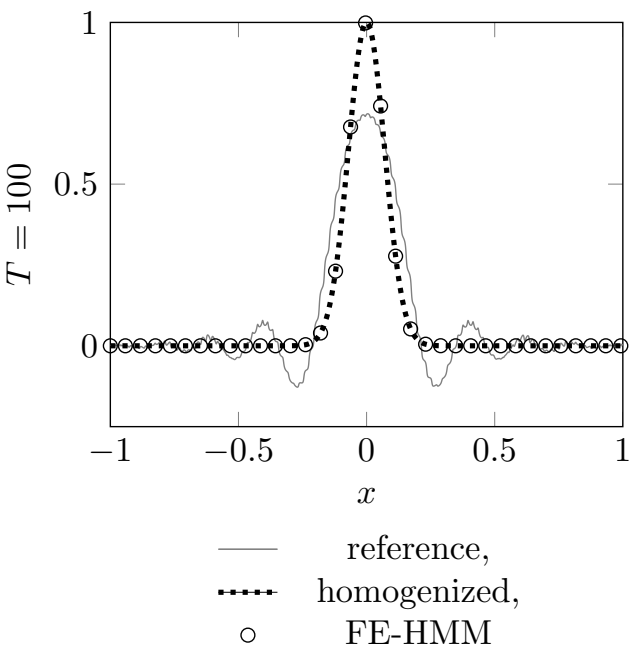

Boussinesq and FE-HMM-L

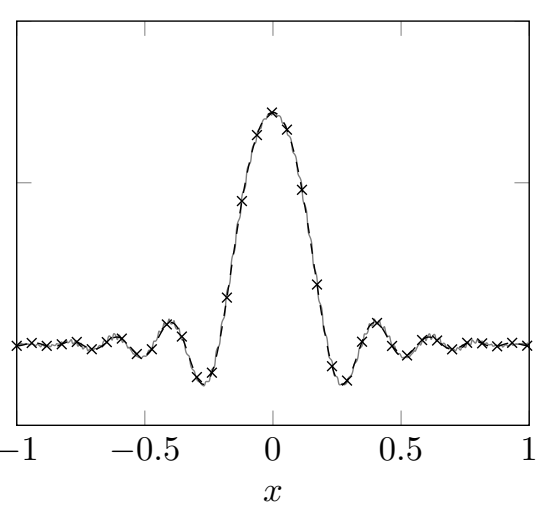

reference,

- - Boussinesq,

$\times \quad$ FE-HMM-L

FIG. 8. One-dimensional periodic medium. Left: The true solution $u^{\varepsilon}$, the solution $u^{0}$ of (2.5), and the FE-HMM scheme from [8] are shown at $T=100$. Right: The true solution $u^{\varepsilon}$, the solution $u^{\mathrm{eff}}$ of the Boussinesq equation (1.3), and the FE-HMM-L solution $u_{H}$ all coincide at $T=100$.

$u^{0}$, as shown in Figure 8. By $T=100$, that is, after 50 revolutions, its amplitude has decreased about $25 \%$, while secondary dispersive wave trains develop. Neither $u^{0}$ nor the FE-HMM solution from [8] recovers those dispersive effects. Here, for the FEHMM and the FE-HMM-L solutions, we use $\mathcal{P}^{3}$ FEs at the macro- and the microscale with $H=2^{-8}$ for improved accuracy. Still, piecewise linear or quadratic FEs could be used just as well, unlike with the FD-HMM from [25, 26], where high-order numerical approximation is necessary at the macroscale.

To underpin the improved long-time accuracy of the Boussinesq equation, we also show in Figure 8 the numerical solution $u^{\mathrm{eff}}$ of (1.3). Both $u_{H}$ and $u^{\mathrm{eff}}$ coincide with the reference solution $u^{\varepsilon}$, even at later times. For this simple purely periodic onedimensional example, the effective coefficients $a^{0}=1$ and $b^{0}=9.09632625 \cdot 10^{-3}$ in (1.3) can be computed with Maple [29]. They are used here only for the numerical approximation of $u^{\mathrm{eff}}$, whereas FE-HMM-L requires no a priori knowledge of any effective quantity.

What if we let time increase even further? To address this question, we compare in Figure $9 u^{\varepsilon}, u^{\mathrm{eff}}$, and $u_{H}$ at times $T=200$ and $T=2000$. While all three still coincide at $T=200$, we observe at the exceedingly large time $T=2000$ how $u^{\varepsilon}$ eventually deviates from the solution of (1.3). The FE-HMM-L and the Boussinesq solutions still coincide. To capture those secondary dispersive effects at exceedingly large times, an even more refined asymptotic analysis would be needed. However, the time frame for the validity of the Boussinesq model and thus also for FE-HMM-L depends not only on $T$, but also on the frequency content of the initial conditions. As shown in Figure 9, if we replace the initial Gaussian pulse with $\sigma^{2}=1 / 100$ by the wider Gaussian with $\sigma^{2}=1 / 20$, both the FE-HMM-L and the Boussinesq solutions still provide reliable approximations of $u^{\varepsilon}$ even at $T=2000$. This behavior is not surprising, since higher frequencies "see" more details of the medium than lower ones, and hence their primary and secondary dispersive effects are stronger. 
Narrow initial Gaussian pulse
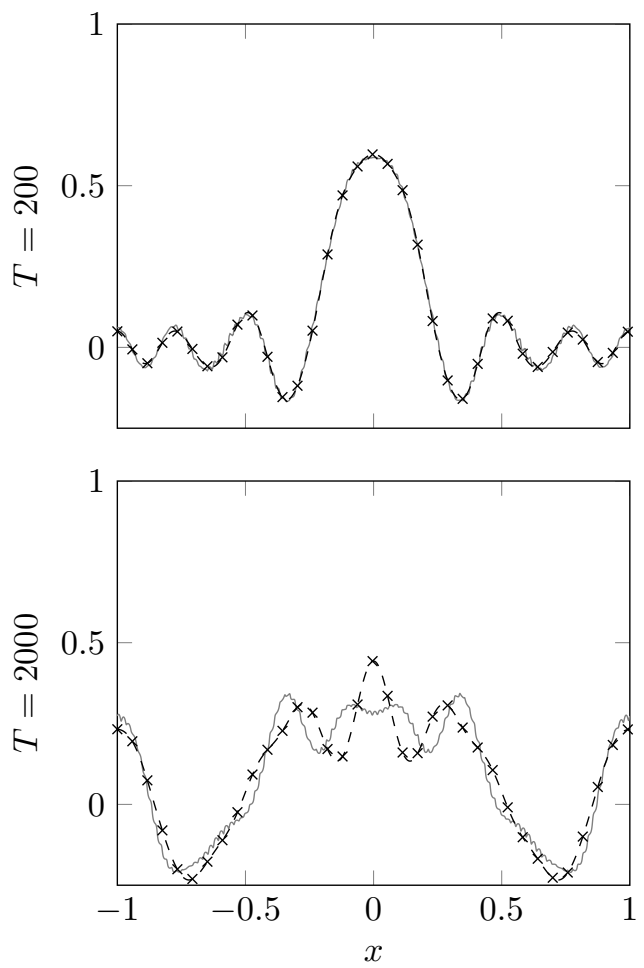

Wide initial Gaussian pulse
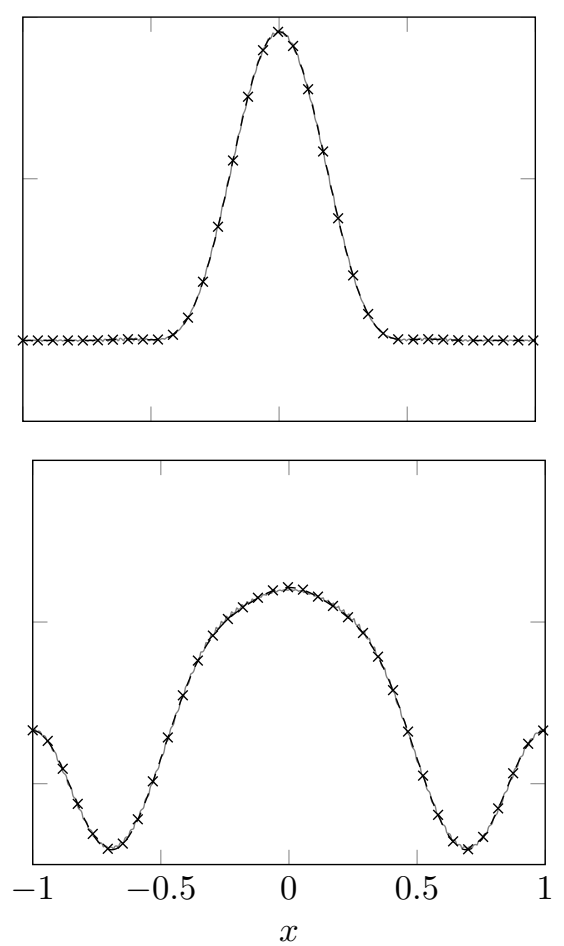

reference, - - Boussinesq, $\times$ FE-HMM-L

FIG. 9. One-dimensional periodic medium. The solutions $u^{\varepsilon}, u^{\mathrm{eff}}$, and $u_{H}$ are shown for two different initial conditions at $T=200$ (top) and $T=2000$ (bottom). Left: Narrow initial Gaussian pulse $\left(\sigma^{2}=1 / 100\right)$. Right: Wide initial Gaussian pulse $\left(\sigma^{2}=1 / 20\right)$. In the lower left frame $u^{\text {eff }}$ and $u_{H}$ coincide, yet both differ from $u^{\varepsilon}$.

Two-dimensional wave guide. Finally, we consider (2.1) with $F \equiv 0$ in a two-dimensional periodic, anisotropic wave guide, $\Omega=[-1,1] \times[0,0.25]$. We impose homogeneous Neumann boundary conditions at the top and bottom boundaries, $x_{2}=$ $0,0.25$, and a periodic boundary condition at the lateral boundaries, $x_{1}=-1,1$. As initial condition, we set $f$ to a Gaussian pulse in the $x_{1}$ direction and $g=0$. Inside the wave guide, the material is anisotropic, and its (squared) velocity tensor is given by

$$
a^{\varepsilon}(x)=\left(\begin{array}{ll}
\sqrt{2}+\sin \left(2 \pi \frac{x_{1}}{\varepsilon}\right) & \\
& 2+\sin \left(2 \pi \frac{x_{1}}{\varepsilon}\right)
\end{array}\right)
$$

with $\varepsilon=1 / 20$. In Figure 10, snapshots of the FE-HMM-L solution $u_{H}$, the fully resolved reference solution $u^{\varepsilon}$, and the FE-HMM solution computed with the scheme from [8] are shown at different times. Both HMM schemes use $\mathcal{Q}^{1}$ FEs with two-point Gauss quadrature for the micro- and the macrodiscretizations, where $H=5 \cdot 10^{-3}$ and $h=5 \cdot 10^{-4}$. With increasing time, the true solution displays a striking dispersive behavior, which is also captured by the FE-HMM-L scheme at the macroscale. In 
$\begin{array}{ll}\text { FE-HMM-L } \quad \text { reference } & \text { FE-HMM }\end{array}$
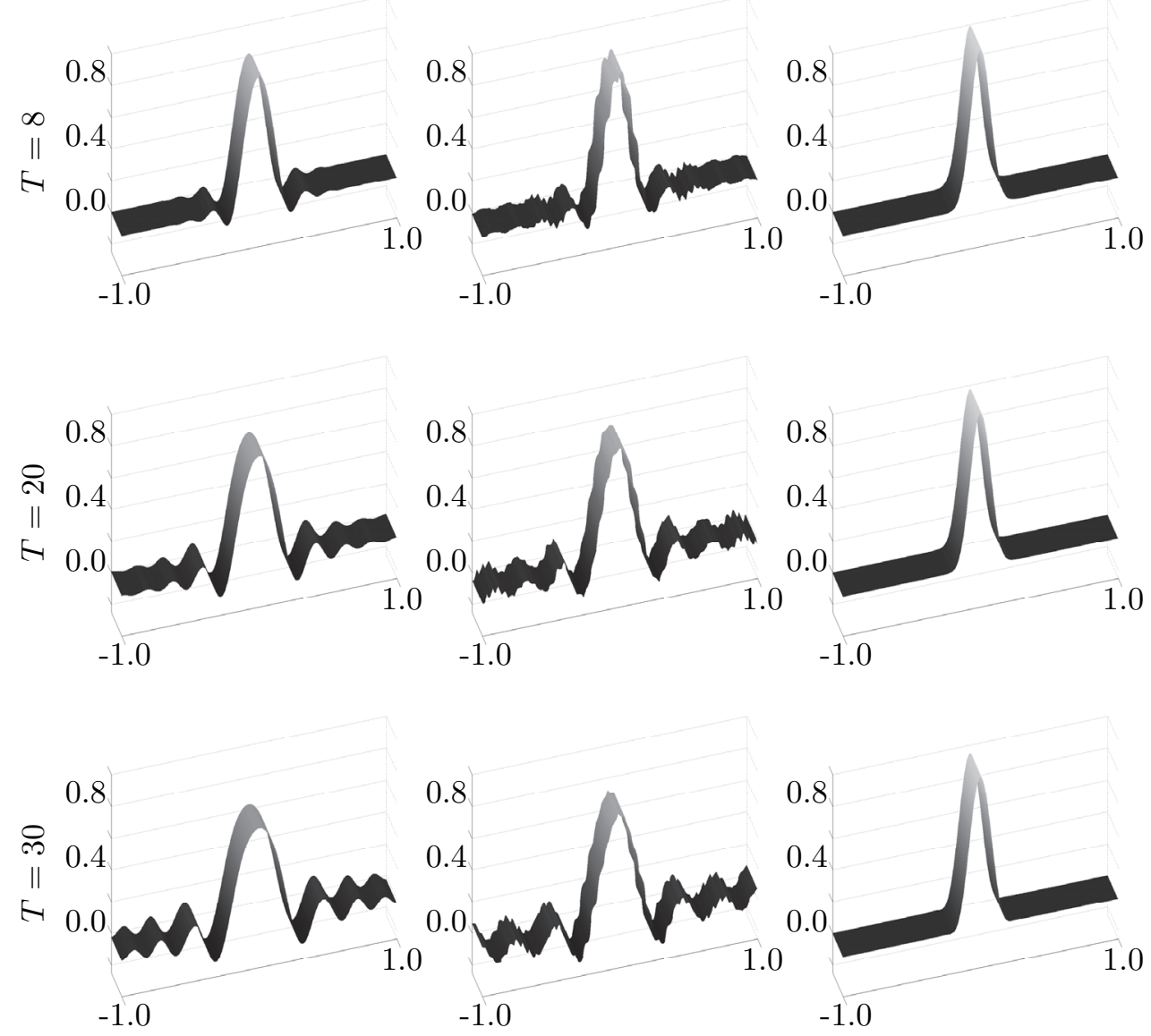

FIG. 10. Two-dimensional wave guide. Snapshots of the FE-HMM-L $u_{H}$ (left), the reference $u^{\varepsilon}$ (middle), and the FE-HMM (right) solutions at times $T=8,20,30$. The physical dispersive effects are correctly captured by the FE-HMM-L but not by the FE-HMM scheme.

contrast, the FE-HMM scheme from [8], as expected, is unable to capture those dispersive effects.

6. Conclusion. We have presented a multiscale FEM for wave propagation in heterogeneous media, which captures not only the short but also the long-time behavior, yet avoids the high computational cost of fully resolved simulations. It is based on an FE discretization of an effective equation at the macroscale, whose a priori unknown coefficients are computed on sampling domains at the microscale within each macro-FE. Optimal error estimates in the energy norm and the $L^{2}$-norm are proved over finite time intervals. They imply convergence to the solution from classical homogenization theory, when both the macro- and the microscale are refined simultaneously, as corroborated by our numerical experiments.

Since the sampling domains themselves scale with the smallest scale, $\varepsilon$, in the problem, the computational work needed for the effective FE-HMM-L stiffness matrix is independent of the fine-scale features of the medium. Moreover, the FE-HMM-L stiffness matrix is computed initially and only once. Then, all subsequent computa- 
tions during the time-stepping procedure occur only on the coarse mesh, while any stability restriction on the time step now depends only on the coarse mesh size $H$. The combined effect of a coarser mesh size with a larger time step yields additional significant savings in computational time, increasingly so at smaller $\varepsilon$.

Because our FE-HMM-L approach leads to a standard Galerkin FE formulation at the macroscale, it immediately applies to higher-dimensional problems, complex geometry, and high-order discretizations. It also easily generalizes to more complicated second-order hyperbolic equations, such as those from electromagnetics or elasticity. The FE-HMM-L method can also be combined with discontinuous Galerkin FE discretizations for the wave equation [5, 28], which provide greater flexibility in the underlying mesh design, waive the need for mass-lumping, and thus lead to inherently parallel fully explicit (local) time-stepping schemes [19].

\section{REFERENCES}

[1] A. AbDulLe, On a priori error analysis of fully discrete heterogeneous multiscale FEM, Multiscale Model. Simul., 4 (2005), pp. 447-459.

[2] A. Abdulle, Analysis of a heterogeneous multiscale FEM for problems in elasticity, Math. Models Methods Appl. Sci., 16 (2006), pp. 615-635.

[3] A. AbDulle, The finite element heterogeneous multiscale method: A computational strategy for multiscale PDEs, in Multiple Scales Problems in Biomathematics, Mechanics, Physics and Numerics, GAKUTO Internat. Ser. Math. Sci. Appl. 31, Gakkōtosho, Tokyo, 2009, pp. $133-181$.

[4] A. AbDulle, A priori and a posteriori error analysis for numerical homogenization: A unified framework, in Multiscale Problems, Ser. Contemp. Appl. Math. CAM 16, Higher Ed. Press, Beijing, 2011, pp. 280-305.

[5] A. AbDulle, Discontinuous Galerkin finite element heterogeneous multiscale method for elliptic problems with multiple scales, Math. Comp., 81 (2012), pp. 687-713.

[6] A. Abdulle And W. E, Finite difference heterogeneous multi-scale method for homogenization problems, J. Comput. Phys., 191 (2003), pp. 18-39.

[7] A. Abdulle, W. E, B. Engquist, and E. Vanden-Eijnden, The heterogeneous multiscale method, Acta Numer., 21 (2012), pp. 1-87.

[8] A. Abdulle and M. J. Grote, Finite element heterogeneous multiscale method for the wave equation, Multiscale Model. Simul., 9 (2011), pp. 766-792.

[9] A. Abdulle, M. J. Grote, and C. Stohrer, FE heterogeneous multiscale method for longtime wave propagation, C. R. Math. Acad. Sci. Paris, 351 (2013), pp. 495-499.

[10] A. Abdulle and C. Schwab, Heterogeneous multiscale FEM for diffusion problems on rough surfaces, Multiscale Model. Simul., 3 (2005), pp. 195-220.

[11] G. A. Baker And V. A. Dougalis, The effect of quadrature errors on finite element approximations for second order hyperbolic equations, SIAM J. Numer. Anal., 13 (1976), pp. 577-598.

[12] A. Bensoussan, J.-L. Lions, and G. Papanicolaou, Asymptotic Analysis for Periodic Structures, AMS Chelsea Publishing, Providence, RI, 2011 (corrected reprint of the 1978 original).

[13] S. Brahim-Otsmane, G. A. Francfort, and F. Murat, Correctors for the homogenization of the wave and heat equations, J. Math. Pures Appl. (9), 71 (1992), pp. 197-231.

[14] Y. Capdeville, L. Guillot, and J.-J. Marigo, 1-D non-periodic homogenization for the seismic wave equation, Geophys. J. Int., 181 (2010), pp. 897-910.

[15] W. Chen And J. Fish, A dispersive model for wave propagation in periodic heterogeneous media based on homogenization with multiple spatial and temporal scales, J. Appl. Mech., 68 (2000), pp. 153-161.

[16] P. G. Ciarlet, The Finite Element Method for Elliptic Problems, Classics Appl. Math. 40, SIAM, Philadelphia, 1978.

[17] P. G. Ciarlet and P.-A. Raviart, The combined effect of curved boundaries and numerical integration in isoparametric finite element methods, in The Mathematical Foundations of the Finite Element Method with Applications to Partial Differential Equations, Academic Press, New York, 1972, pp. 409-474.

[18] D. Cioranescu and P. Donato, An Introduction to Homogenization, Oxford Lecture Ser. Math. Appl. 17, The Clarendon Press, Oxford University Press, New York, 1999. 
[19] J. Diaz And M. J. Grote, Energy conserving explicit local time stepping for second-order wave equations, SIAM J. Sci. Comput., 31 (2009), pp. 1985-2014.

[20] T. Dohnal, A. Lamacz, And B. Schweizer, Dispersive Effective Equations for Waves in Heterogeneous Media on Large Time Scales, Preprint 2013-01, Technische Universität, Dortmund, Dortmund, Germany, 2013; available online at http://www.mathematik. tu-dortmund.de/lsi/lamacz/mathematicalPreprint-2013-01DispMulti.pdf.

[21] Q.-L. Dong AND L.-Q. CAO, Multiscale asymptotic expansions and numerical algorithms for the wave equations of second order with rapidly oscillating coefficients, Appl. Numer. Math., 59 (2009), pp. 3008-3032.

[22] W. E And B. Engquist, The heterogeneous multiscale methods, Commun. Math. Sci., 1 (2003), pp. 87-132.

[23] W. E, P. Ming, And P. Zhang, Analysis of the heterogeneous multiscale method for elliptic homogenization problems, J. Amer. Math. Soc., 18 (2005), pp. 121-156.

[24] B. Engquist, H. Holst, And O. Runborg, Multiscale methods for the wave equation, PAMM, 7 (2007), pp. 1140903-1140904.

[25] B. Engquist, H. Holst, And O. Runborg, Multi-scale methods for wave propagation in heterogeneous media, Commun. Math. Sci., 9 (2011), pp. 33-56.

[26] B. Engquist, H. Holst, And O. Runborg, Multiscale methods for wave propagation in heterogeneous media over long time, in Numerical Analysis of Multiscale Computations, Lect. Notes Comput. Sci. Eng. 82, B. Engquist, O. Runborg, and Y.-H. R. Tsai, eds., SpringerVerlag, Berlin, Heidelberg, 2012, pp. 167-186.

[27] L. C. Evans, Partial Differential Equations, Grad. Stud. Math. 19, AMS, Providence, RI, 1998.

[28] M. J. Grote, A. Schneebeli, and D. Schötzau, Discontinuous Galerkin finite element method for the wave equation, SIAM J. Numer. Anal., 44 (2006), pp. 2408-2431.

[29] H. Holst, Algorithms and Codes for Wave Propagation Problems, Technical report, KTH Royal Institute of Technology, School of Computer Science and Communication, Stockholm, Sweden, 2011.

[30] A. Lamacz, Dispersive effective models for waves in heterogeneous media, Math. Models Methods Appl. Sci., 21 (2011), pp. 1871-1899.

[31] J.-L. Lions And E. Magenes, Problèmes aux limites non homogènes et applications, Vol. 1, Dunod, Paris, 1968.

[32] H. Owhadi And L. Zhang, Numerical homogenization of the acoustic wave equations with a continuum of scales, Comput. Methods Appl. Mech. Engrg., 198 (2008), pp. 397-406.

[33] P.-A. RAVIART, The use of numerical integration in finite element methods for solving parabolic equations, in Topics in Numerical Analysis, Academic Press, London, 1973, pp. 233-264.

[34] F. Santosa And W. W. Symes, A dispersive effective medium for wave propagation in periodic composites, SIAM J. Appl. Math., 51 (1991), pp. 984-1005.

[35] T. Vdovina, S. E. Minkoff, and O. Korostyshevskaya, Operator upscaling for the acoustic wave equation, Multiscale Model. Simul., 4 (2005), pp. 1305-1338.

Copyright (c) by SIAM. Unauthorized reproduction of this article is prohibited. 